UNIVERSIDADE FEDERAL DO RIO GRANDE DO SUL

INSTITUTO DE INFORMÁTICA

PROGRAMA DE PÓS-GRADUAÇÃO EM COMPUTAÇÃO

GERALDO FRANCISCO DE OLIVEIRA JUNIOR

\title{
A Generic Processing in Memory Cycle Accurate Simulator under Hybrid Memory \\ Cube Architecture
}

Dissertation presented in partial fulfillment of the requirements for the degree of

Master of Computer Science

Advisor: Prof. Dr. Luigi Carro

Porto Alegre

September 2017 


\section{CIP - CATALOGING-IN-PUBLICATION}

de Oliveira Junior, Geraldo Francisco

A Generic Processing in Memory Cycle Accurate Simulator under Hybrid Memory Cube Architecture / Geraldo Francisco de Oliveira Junior. - Porto Alegre: PPGC da UFRGS, 2017.

70 f.: il.

Thesis (Master) - Universidade Federal do Rio Grande do Sul. Programa de Pós-Graduação em Computação, Porto Alegre, BRRS, 2017. Advisor: Luigi Carro.

1. In-Memory Processing. 2. Simulators. 3. Hybrid Memory Cube. 4. 3D-Stacked. I. Carro, Luigi. II. Título.

UNIVERSIDADE FEDERAL DO RIO GRANDE DO SUL

Reitor: Prof. Rui Vicente Oppermann

Vice-Reitora: Prof ${ }^{\mathrm{a}}$. Jane Fraga Tutikian

Pró-Reitor de Pós-Graduação: Prof. Celso Giannetti Loureiro Chaves

Diretora do Instituto de Informática: Prof ${ }^{\mathrm{a}}$. Carla Maria Dal Sasso Freitas

Coordenador do PPGC: Prof. João Luiz Dihl Comba

Bibliotecária-chefe do Instituto de Informática: Beatriz Regina Bastos Haro 
"The fairest thing we can experience is the mysterious."

- Albert Einstein 


\section{AGRADECIMENTOS}

Agradeço aos meus pais pelo suporte. Agradeço ao Paulo pelo apoio durante a elaboração deste trabalho (e por todas as discussões). Agradeço ao Professor Luigi por me ensinar a pensar fora da caixinha. Agradeço aos amigos do Laboratório de Sistemas Embarcados. Em especial ao Marcelo e o Anderson pelas caronas. O Jeckson pela parceria. O Arthur pelas histórias. Agradeço ao Tiago pelas longas discussões sobre Game of Thrones. E ao Leonardo por ser uma enciclopédia. Agradeço ao Professor Caco pelos concelhos. E agradeço a Michele por aguentar minhas bagunças. 


\begin{abstract}
PIM - a technique which computational elements are added close, or ideally, inside memory devices - was one of the attempts created during the 1990s to try to mitigate the memory wall problem. Nowadays, with the maturation of 3D integration technologies, a new landscape for novel PIM architectures can be investigated. To exploit this new scenario, researchers rely on software simulators to navigate throughout the design evaluation space. Today, most of the works targeting PIM implement in-house simulators to perform their experiments. However, this methodology might hurt overall productivity, while it might also preclude replicability. In this work, we showed the development of a precise, modular and parametrized PIM simulation environment. Our simulator, named CLAPPS, targets the HMC architecture, a popular 3D-stacked memory widely employed in state-of-the-art PIM accelerators. We have designed our mechanism using the SystemC programming language, which allows native parallel simulation. The primary contribution of our work lies in developing a user-friendly interface to allow easy PIM architectures exploitation. To evaluate our system, we have implemented a PIM module that can perform vector operations with different operand sizes using the proposed set of tools.
\end{abstract}

Keywords: In-Memory Processing. Simulators. Hybrid Memory Cube. 3D-Stacked. 


\title{
Um Simulador Genérico Ciclo-Acurado para Processamento em Memória baseado na arquitetura da Hybrid Memory Cube
}

\begin{abstract}
RESUMO
PIM - uma técnica onde elementos computacionais são adicionados perto, ou idealmente, dentro de dispositivos de memória - foi uma das tentativas criadas durante os anos 1990 visando mitigar o notório memory wall problem. Hoje em dia, com o amadurecimento do processo de integração 3D, um novo horizonte para novas arquiteturas PIM pode ser explorado. Para investigar este novo cenário, pesquisadores dependem de simuladores em software para navegar pelo espaço de exploração de projeto. Hoje, a maioria dos trabalhos que focam em PIM, implementam simuladores locais para realizar seus experimentos. Porém, esta metodologia pode reduzir a produtividade e reprodutibilidade. Neste trabalho, nós mostramos o desenvolvimento de um simulador de PIM preciso, modular e parametrizável. Nosso simulador, chamado CLAPPS, visa a arquitetura de memória $H M C$, uma memória 3D popular, que é amplamente utilizada em aceleradores PIM do estado da arte. Nós desenvolvemos nosso mecanismo utilizando a linguagem de programação SystemC, o que permite uma simulação paralela nativamente. A principal contribuição do nosso trabalho se baseia em desenvolver a interface amigável que permite a fácil exploração de arquiteturas PIM. Para avaliar o nosso sistema, nós implementamos um modulo de PIM que pode executar operações vetoriais com diferente tamanhos de operandos utilizando o proposto conjunto de ferramentas.
\end{abstract}

Palavras-chave: Processamento em Memória, Simuladores, Hybrid Memory Cube. 


\section{LIST OF ABBREVIATIONS AND ACRONYMS}

\begin{tabular}{|c|c|}
\hline $\mathrm{ACM}$ & Active Memory Cube \\
\hline $\mathrm{AF}$ & Atomic Flag \\
\hline ALU & Arithmetic Logic Unit \\
\hline CGRA & Coarse-Grain Reconfigurable Array \\
\hline CLAPPS & Cycle Accurate Parallel PIM Simulator \\
\hline ConvNet & Convolutional Neural Network \\
\hline CRC & Cycle Redundancy Check \\
\hline DDR & Double Data Rate \\
\hline DIMM & Dual in-line Memory Module \\
\hline DINV & Data Invalid \\
\hline DRAM & Dynamic Random Access Memory \\
\hline ERRSTAT & Error Status \\
\hline FRP & Forward Retry Pointer \\
\hline FSM & Finite State Machine \\
\hline $\mathrm{FU}$ & Functional Unit \\
\hline GPU & Graphics Processing Unit \\
\hline HBM & High Bandwidth Memory \\
\hline HDL & Hardware Description Language \\
\hline HIVE & HMC Instruction Vector Extensions \\
\hline HMC & Hybrid Memory Cube \\
\hline HPC & High Performance Computing \\
\hline ISA & Instruction Set Architecture \\
\hline LM & Link Master \\
\hline LS & Link Slave \\
\hline
\end{tabular}




$\begin{array}{ll}\text { NDP } & \text { Near-Data Processing } \\ \text { NN } & \text { Neural Network } \\ \text { NoC } & \text { Network-on-Chip } \\ \text { Pb } & \text { Poison bit } \\ \text { PIM } & \text { Processor-in-Memory } \\ \text { RRP } & \text { Return Retry Pointer } \\ \text { RTC } & \text { Return Token Count } \\ \text { RTL } & \text { Register Transfer Level } \\ \text { RVU } & \text { Reconfigurable Vector Unit } \\ \text { SEQ } & \text { Sequence Number } \\ \text { SLID } & \text { Source Link ID } \\ \text { SMC } & \text { Smart Memory Cube } \\ \text { SoC } & \text { System-on-Chip } \\ \text { SSD } & \text { Solid-State Drive } \\ \text { SST } & \text { Structural Simulation Toolkit } \\ \text { TSV } & \text { Through-Silicon Via } \\ & \end{array}$




\section{LIST OF FIGURES}

Figure 1.1 Current memory and CPU scaling trends. The processor line depicts the number of cores per socket, while the memory line predicts the memory capacity per socket.

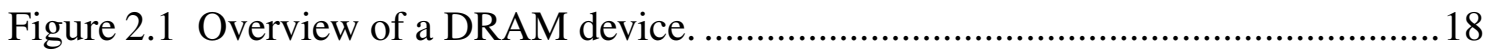

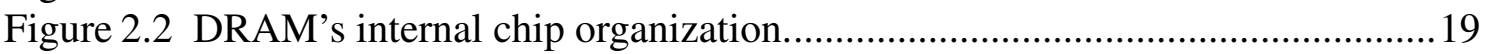

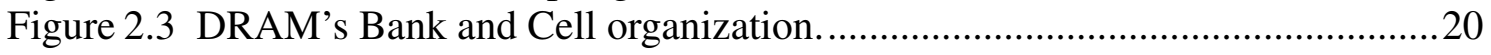

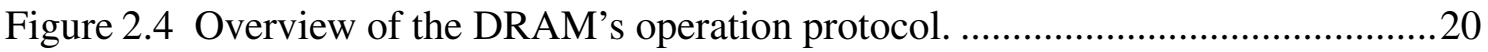

Figure 2.5 Overview of the Hybrid Memory Cube (HMC) organization. In this figure, a Partition is equivalent to a Dynamic Random Access Memory (DRAM)'s

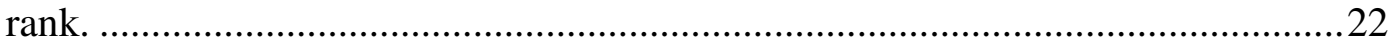

Figure 2.6 Fields for request and response HMC packets. .......................................23

Figure 2.7 Overview of the Transceiver module organization.....................................25

Figure 2.8 Representation of the low-interleave algorithm used as memory map-

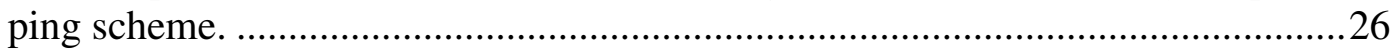

Figure 4.1 Simplified packet diagram of the proposed simulator. ….............................35

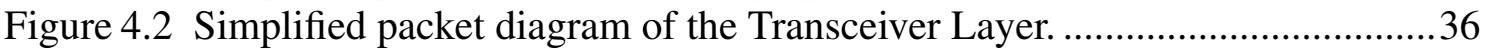

Figure 4.3 Block diagram of the packet generator module.......................................... 37

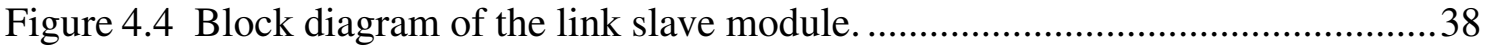

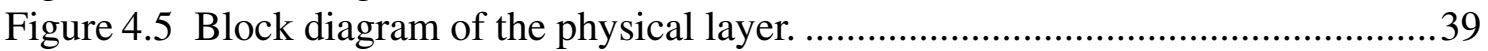

Figure 4.6 Block diagram of the link slave module ......................................................... 41

Figure 4.7 Simplified packet diagram of the Vault Layer. ............................................. 42

Figure 4.8 Block diagram of the Vault Request module.............................................43

Figure 4.9 Block diagram of the Memory and TSV controller modules. .......................47

Figure 4.10 Block diagram of native PIM module....................................................4

Figure 4.11 Block diagram of the Vault Response module.........................................48

Figure 4.12 Block diagram of the proposed PIM Interface. .........................................51

Figure 4.13 Block diagram of the Traffic Monitor class...............................................53

Figure 5.1 Simulation steps our mechanism travels through execution........................56

Figure 6.1 Link and Vault total bandwidth for sequential read and write requests. ......58

Figure 6.2 Vault total bandwidth for sequential read requests. ..................................58

Figure 6.3 Link total bandwidth for sequential read requests. ..................................59

Figure 6.4 Vault total bandwidth for sequential and random atomic requests. ..............60

Figure 6.5 Link total bandwidth for sequential and random atomic requests. ...............60

Figure 6.6 Overview of the Reconfigurable Vector Unit (RVU) organization. ..............61

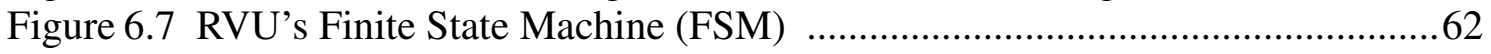




\section{LIST OF TABLES}

Table 1.1 Processor-in-Memory (PIM) works and their simulators...............................15

Table 2.1 Programming languages categorized targeting distinct levels of abstraction..28

Table 3.1 Comparison between available PIM simulators. "?" indicates the infor-

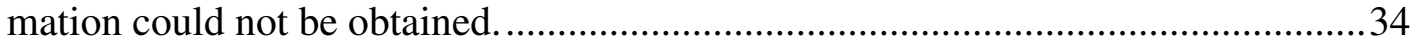

Table 4.1 Description of th request_decoder module, its input/output ports, meth-

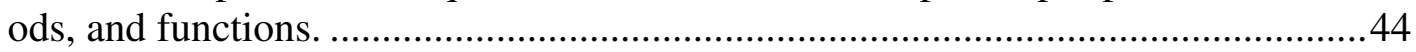

Table 4.2 Description of th response_control module, its input/output ports, meth-

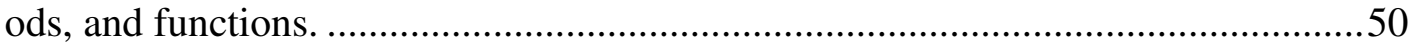

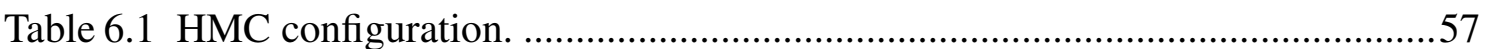

Table 7.1 Comparison between available PIM simulators and CLAPPS. "?" indicates the information could not be obtained. 


\section{CONTENTS}

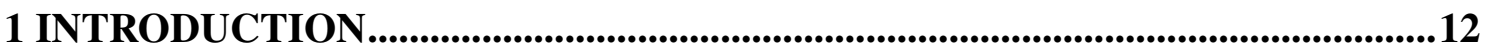

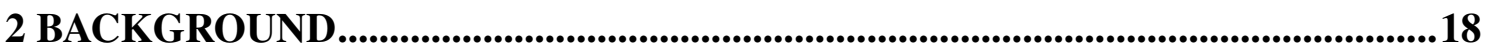

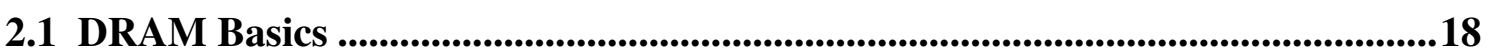

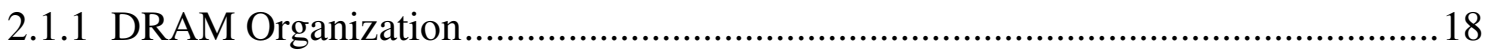

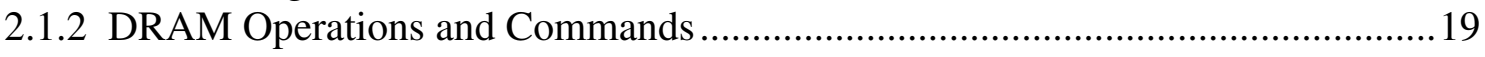

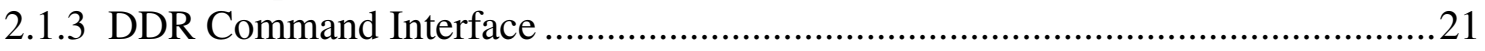

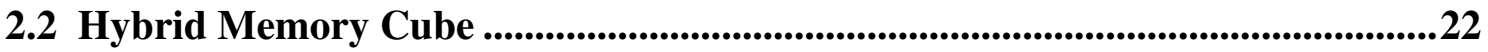

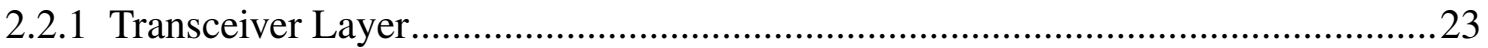

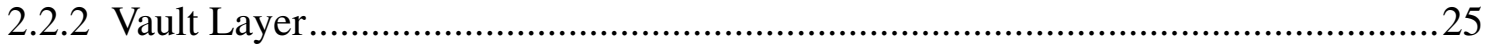

2.3 The SystemC Programming Model ...............................................................................27

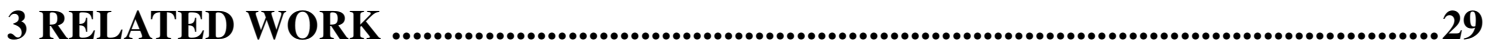

3.1 Processing-in-Memory Architectures...................................................................29

3.2 Processing-in-Memory Simulators ........................................................................................32

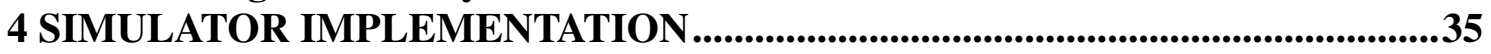

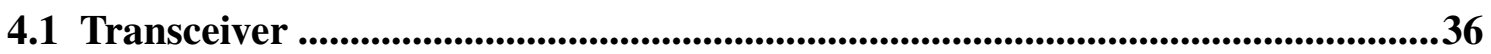

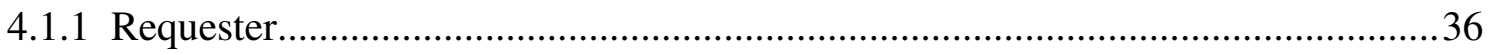

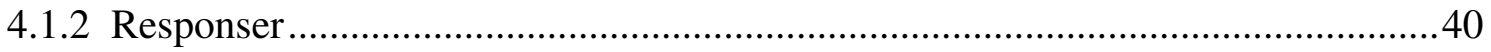

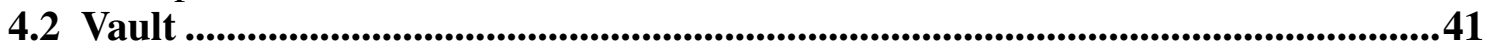

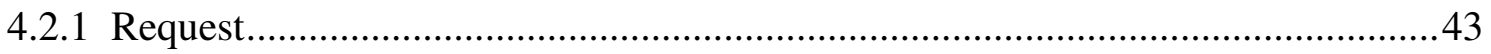

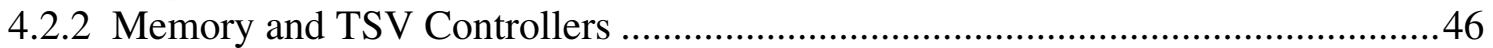

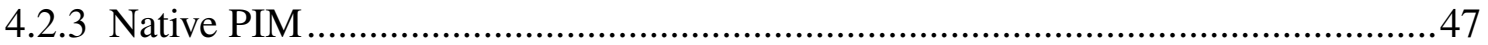

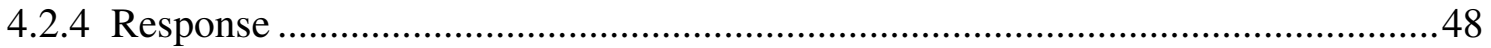

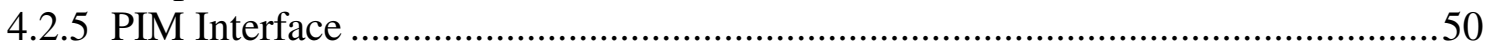

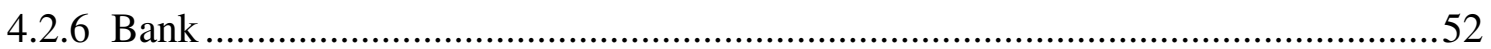

4.3 Traffic Monitor .......................................................................................................54

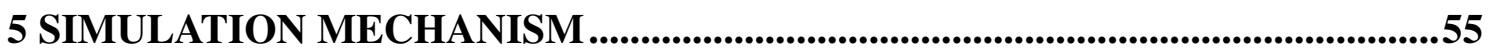

6 EXPERIMENTAL SETUP AND RESULTS..........................................................57

6.1 Memory Validation .......................................................................................................57

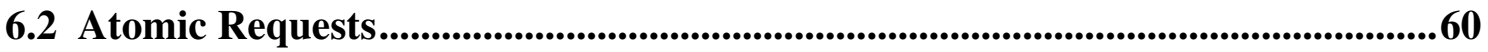

6.3 Case of Study: PIM Interface .........................................................................................661

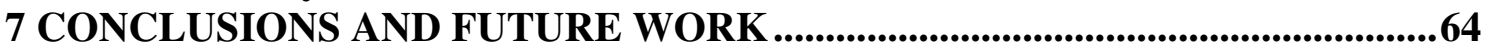

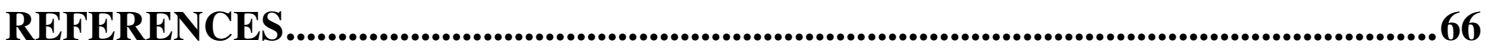




\section{INTRODUCTION}

For decades, the slow advancements of main memory technology and manufacturing processes have been overshadowed by Moore's law (SCHALLER, 1997). As smaller transistors paved the way for ever-faster processing units, the same could not be done for memory devices, which have different trade-offs and design points. This led to both a performance and a scaling gap between processing units and memory devices (WULF; MCKEE, 1995). Figure 1.1, extracted from (LIM et al., 2009), illustrates the current trend related to memory and CPU scaling. One can notice that the number of CPU cores per socket has been doubling every two years, while the memory capacity doubles every three years. If future systems continue to scale in this fashion, we expect memory capacity to start dropping by $30 \%$ every two years (LIM et al., 2009).

The bottleneck that the memory hierarchy represents to modern computational systems is due to two fundamental reasons:

1. Technological. Dynamic Random Access Memorys (DRAMs) are commonly employed as the main memory device because of its reasonable cost per bit since it requires only one transistor to store a single bit (JACOB; NG; WANG, 2010). As detailed in Section 2.1, DRAM devices are charge-based modules, meaning that data is stored using capacitor cells. This technological design choice adds com-

Figure 1.1: Current memory and CPU scaling trends. The processor line depicts the number of cores per socket, while the memory line predicts the memory capacity per socket.

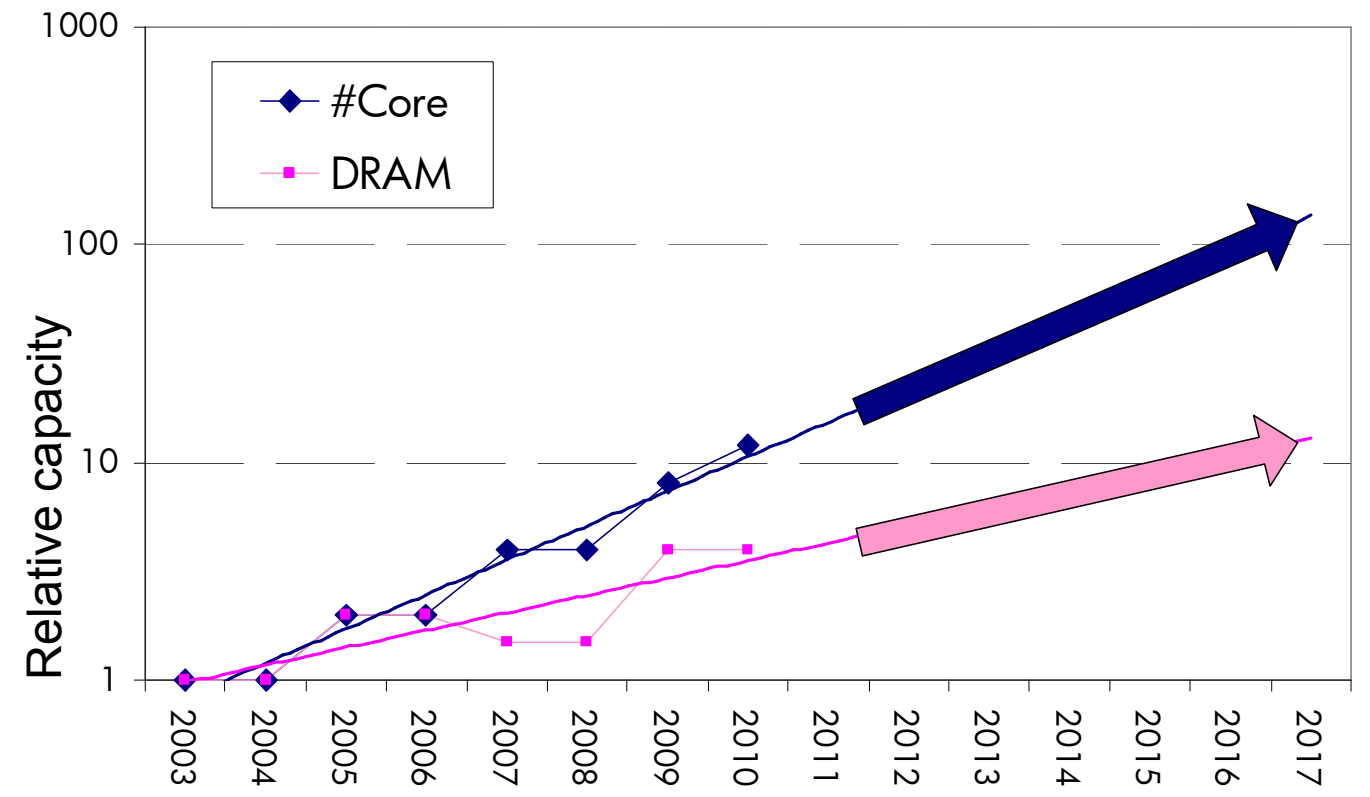

Source: (LIM et al., 2009). 
plexity and extra latency to access memory because (i) capacitors leak charge over time, forcing data to be refreshed during fixed time intervals, and (ii) all read operations are destructive; therefore all read commands need to be followed by a write command.

2. Architectural. The memory system has always been treated as a slave mechanism that only responds to read/write commands. However, as previous works have shown (LEE et al., 2010; STUECHELI et al., 2010), performance can be improved when the memory system and computational units cooperate sharing run-time information. For instance, it is possible to improve memory throughput by (i) taking advantage of memory parallelism and (ii) building memory controllers that explore data locality.

The gap between memory and CPU technology becomes even more visible in systems that process large volumes of data (MURPHY, 2007; RADULOVIC et al., 2015; LIM et al., 2009), in the so-called Big Data and High Performance Computing (HPC) workloads. For example, in a data-center scenario, a single query may request massive amounts of data to be moved between the whole memory system. Passing through non-volatile to the main memory modules, then by several layers of cache memories, until the requested data finally reaches the processor, where typically just a small amount of computation will be performed (SANTOS et al., 2017). Even though this issue is becoming more problematic in modern workstations, (WULF; MCKEE, 1995) has first foreseen the memory impact for future systems in 1995, calling it the Memory Wall Problem. The authors observed that processor speed rises at a range of $75 \%$ per year, while memory speed increases by a factor of $7 \%$ from each generation. This enormous gap between memory and CPU performance implies that the memory system would be the primary source of performance slowdowns. These days, to access main memory, the processor has to wait for around 100 clock cycles to receive the requested data back from memory. This scenario is even more problematic when one considers multi-core and heterogeneous systems, where multiple memory requests are being made simultaneously, thus adding extra latency due to conflicted accesses (MOSCIBRODA; MUTLU, 2007; EBRAHIMI et al., 2010; SUBRAMANIAN et al., 2013). Besides that, current DRAM memories are not able to supply enough bandwidth for HPC and Big Data applications (RADULOVIC et al., 2015). To conclude, what we have today are systems that require significant bandwidth from the memory system, but a memory system that cannot provide such amount of data per seconds. 
Endeavoring to reduce the memory wall impact over large workloads, several memory manufacturers have taken advantage of 3D-stacked integration technology to implement 3D-stacked memories that fit better to HPC workloads. In a 3D-stack environment, several layers of memory modules are vertically connected by Through-Silicon Via (TSV), improving data density and also increasing memory bandwidth. Also, 3D integration makes it possible to implement heterogeneous stacks, with layers of memories and logic all together in one single chip. These features help to reduce the latency to access memory by moving memory controllers from CPU units into the memory device itself. Some commercial 3D stacked memories are available in the market nowadays, as Hybrid Memory Cube (HMC) (Hybrid Memory Cube Consortium, 2013), High Bandwidth Memory (HBM) (HONG, 2014), and DiRAM4 (Tezzaron, 2015).

Most important, the possibility to combine memory devices and computation elements into one single chip renews the prospect to explore Processor-in-Memory (PIM) architectures (PUGSLEY et al., 2014). PIM, previously named Near-Data Processing (NDP), was first designed to reduce the execution time of memory bounded applications by placing logic elements inside DRAM modules, aiming to explore their much larger internal bandwidth (PATTERSON et al., 1997). However, besides improving how the system would exploit memory bandwidth, PIM approaches can potentially help reduce overall memory access latency. Moreover, by adding logic modules closer to the memory, the amount of data that needs to navigate throughout the whole memory system can be reduced, therefore diminishing the total number of energy consumed by the entire system - a key design constraint for large-scale data-processing centers and embedded systems.

To understand the impact that memory components impose to a system, either regarding performance or energy consumption, researchers in academia heavily rely on software simulators to navigate throughout the design evaluation space. A lot of effort has been made during the past years by the academia to build memory simulators that could push state-of-the-art designs to the next level of complexity. To list some, the DRAMSim2 simulator (ROSENFELD; COOPER-BALIS; JACOB, 2011), a cycle-accurate Double Data Rate (DDR) memory simulator, is widely employed to estimate DDR2/3 speed and power consumption; the Cacti (SHIVAKUMAR; JOUPPI, 2001) model is a popular tool to estimate cache performance, area, and power consumption; and VSSIM (YOO et al., 2013) is a new Solid-State Drive (SSD) simulator that can mimic today's SSD architectures.

Since PIM has emerged again recently, simulating the industry's new memory de- 
Table 1.1: PIM works and their simulators.

\begin{tabular}{lll}
\hline PIM Architecture & Platform & Category \\
\hline Tesseract (AHN et al., 2015a) & In-house & cycle-accurate \\
IMPICA (HSIEH et al., 2016) & gem5 & cycle-accurate \\
NDA (FARMAHINI-FARAHANI et al., 2015) & gem5 & cycle-accurate \\
SMC (AZARKHISH et al., 2016a) & gem5 & cycle-accurate \\
AMC (SURA et al., 2015) & Mambo Simulator & cycle-accurate \\
HIVE (ALVES et al., 2016) & SiNuca & functional \\
GPU+CPU (XU; ZHANG; JAYASENA, 2015) & In-house & analytical \\
Neurostream (AZARKHISH et al., 2017) & In-house & cycle-accurate \\
NIM (OLIVEIRA et al., 2017) & SiNuca & functional \\
RVU(SANTOS et al., 2017) & SiNuca & functional \\
\hline
\end{tabular}

Source: Provided by the author.

vices or even testing novel PIM mechanisms is a common issue that researchers face. As illustrated in Section 3, most of the works that target PIM implement in-house simulators to support their experiments (ALVES et al., 2016; XU; ZHANG; JAYASENA, 2015; AHN et al., 2015b; OLIVEIRA et al., 2017; SANTOS et al., 2017). Table 1.1 classifies some previous PIM works and the simulator used in each case. Today's PIM simulators can be categorized into three broad groups: functional, cycle-accurate, and analytical models. To illustrate, one could find works as (SANTOS et al., 2017), (OLIVEIRA et al., 2017), and (ALVES et al., 2016) that make use of simulators in the functional category. These simulators can determine the device performance isolated from other system components while modeling none or a limited number of elements from the device's micro-architecture. On the other hand, simulators as the ones employed by (FARMAHINI-FARAHANI et al., 2015) and (AZARKHISH et al., 2016a) perform a cycle-by-cycle evaluation of all system components, from interconnection modules to the operational system. Finally, the simulator used by (XU; ZHANG; JAYASENA, 2015) fits in the third class of simulators. Their simulator is designed using machine learning techniques to estimate the final device performance.

Two problems can be foreseen out of this scenario of multiple PIM simulators. First, a significant part of researching effort is spent building the required simulation environment. Second, it becomes difficult to reproduce another researchers' work. Another well-known problem related to PIM simulators is how to measure three important aspects of embedded system design: area, power, and energy consumption. Evaluating the total 
design area is important to put boundaries to PIM architectures. Also, dissipated power and energy consumption are two key design parameters in both embedded and HPC environments. Some approaches can be employed to obtain those metrics. First, one could build their design model using Hardware Description Language (HDL) and directly extract the produced circuitry using synthesis tools (AZARKHISH, 2016). Even though the Register Transfer Level (RTL) model may produce the most accurate result, its design flow is extremely time-consuming. Another approach would be adopting tools as McPat (LI et al., 2013) to estimate the model outputs. However, it is possible that the estimation tool may produce imprecise results due the number of variables related to the matter (XI et al., 2015).

Goal. In this work, we aim to build a generic PIM simulator that can aid researcher to build and investigate novel PIM mechanisms. However, there are two major challenges we have to deal with to cope with this task.

Challenge 1. Which type of 3D-memory organization should our simulator target? Since the maturation of the 3D manufacturing technology, several stacking memories devices have been proposed both by the academia and by different vendors (HONG, 2014; Hybrid Memory Cube Consortium, 2013; Tezzaron, 2015). However, in most cases, there is not a detailed documentation public available that describes these devices.

Challenge 2. How to provide a simple mechanism to allow designs to implement new PIM architectures easily? An ideal PIM simulator would have to provide to the final user two critical capabilities. First, it needs to be accurate as a memory simulator. Second, the simulator needs to be flexible and easy to use. It needs to be flexible to be employed in different PIM designs, which would probably use different types and numbers of Functional Units (FUs), internal storage elements, distinct and specialized Instruction Set Architectures (ISAs), and target various classes of applications. Also, the simulator needs to be easy to use to reduce the amount of effort that is spent understanding and extending the simulator.

To solve the first challenge, we have decided to employ the HMC device as our 3D-stacking memory mechanism. We took this decision based on two major facts. First, even though there is no documentation publicly available describing in details the internals of an HMC module, the HMC Consortium has published an extensive documentation that provides a high-level view of the memory organization and employed communication's protocols. Second, there are a large number of works that have investigated the capabilities and trade-offs related to HMC devices (ROSENFELD, 2014; AZARKHISH 
et al., 2016a; MATHEW et al., 2017).

To solve the second challenge, we have implemented into our simulator a PIM interface that deals with conventional operations, as reads and writes, to the user. In this way, the user can focus only on designing the internals of their mechanism. Also, we have extended the HMC communication's protocol to understand a new set of operations without imposing significant modifications to the already proposed mechanism.

Contributions. The major contributions of this work are:

- We built a generic Cycle Accurate Parallel PIM Simulator (CLAPPS) that can be used to create custom PIM architectures. Our framework, described in details in Section 4, has been developed using the SystemC programming language (PANDA, 2001). We have chosen to develop our system using SystemC because it can be easily integrated with already available simulation platforms, for example, with the widely employed gem5 (BINKERT et al., 2011) simulator ${ }^{1}$. Also, a SystemC module can quickly produce a synthesizable RTL model. Moreover, by implementing our simulation using SystemC, we were able to simulate parallel behavior natively. In the current version, we developed an HMC simulator targeting its latest technical specifications (Hybrid Memory Cube Consortium, 2013), including all HMC instructions.

- We validated the memory side of our simulator using previous works that target HMC devices. In special, we have used the work of (ROSENFELD, 2014) as a guide for the results expected of internal and external memory bandwidth. To demonstrate the PIM capabilities of our simulator, we have implemented a simple vectorial processor, similar to the one proposed by (SANTOS et al., 2017), using the proposed PIM interface (Section 6).

- With our simulator, we could observe common challenges that new PIM architectures would possibly have to deal in the future, as the role of a 3D-stacking memory in the system, programmability, and data coherence (Section 7).

\footnotetext{
${ }^{1} \mathrm{Gem} 5$ is a robust and extensible system that can simulate most elements of a computer system, including a number of instruction sets, micro architecture organizations, memory devices, interconnections, and communication protocols.
} 


\section{BACKGROUND}

In this section, we introduce some basic concepts that will be used throughout the rest of this work. First, we give a brief explanation about the Dynamic Random Access Memory (DRAM) architecture, organization, and functionalities. For a more detailed overview of DRAM devices, we refer the reader to (JACOB; NG; WANG, 2010; HASSAN et al., 2017; PATEL; KIM; MUTLU, 2017; SESHADRI et al., 2013; LIU et al., 2013; LIU et al., 2012; LEE et al., 2015). Second, we describe in details the internal structure and the communication's protocol of an Hybrid Memory Cube (HMC) module. All information related to HMC devices was obtained from the latest HMC specification (Hybrid Memory Cube Consortium, 2013). Finally, we present a brief description of the SystemC programming model.

\subsection{DRAM Basics}

\subsubsection{DRAM Organization}

Figure 2.1 illustrates a high-level view of a DRAM module. A single DRAM device is organized hierarchically into Channels, Dual in-line Memory Modules (DIMMs), Ranks, Chips, Banks, and Cells. A Channel receives commands, address, and data via buses connected to the memory controller placed into the CPU socket. Each Channel has one or more DIMMs. Each DIMM is composed of a set of one or more Ranks, and operates independently of each other. In today's architectures, a single memory controller can operate over a single Channel or over multiple Channels using a wider I/O interface. A Rank consists of a set of DRAM's Chips that share the command signals and works in a lockstep. Typically there are from four to eight DRAM's Chips in a Rank. Each Chip

Figure 2.1: Overview of a DRAM device.

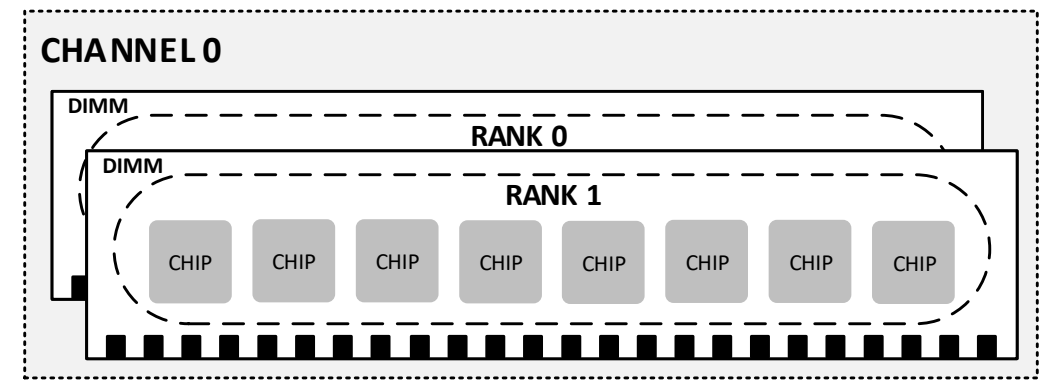

Source: Provided by the author. 
Figure 2.2: DRAM's internal chip organization.

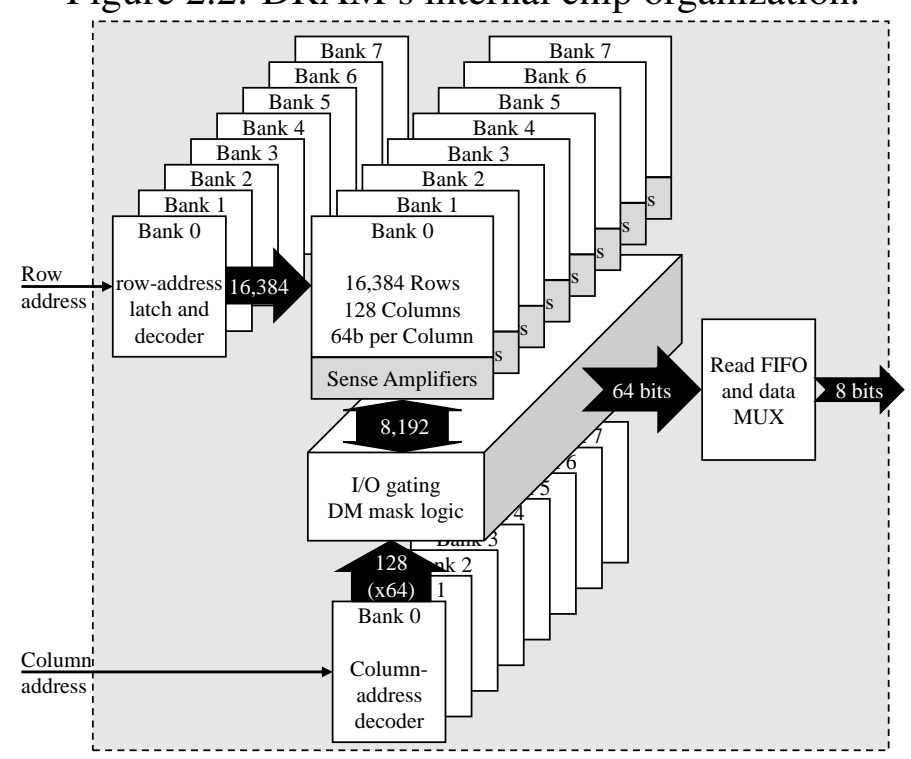

Source: (SANTOS et al., 2016).

contributes to a portion of the data that flows from/to the request device. For example, in a system with a Channel I/O interface of 64 bits, and 8 Chips, each Chip would output 8 bits of data in parallel.

Figure 2.2 depicts the internals of a single DRAM Chip. The primary block in a Chip is the Bank. Inside a single Chip are usually four to eight Banks that can be accessed independently and operate in parallel. Besides the Banks, there are a set of latches and decoders used to access the Banks' rows and columns, and some logic elements used to output data to the bus interface. Figure 2.3a illustrates the internal organization of a DRAM Bank. Each Bank is composed of a 2D grid of Cells, as shown in Figure 2.3b. Each row of Cells is connected via a wordline, and each column of Cells is connected via a bitline. Also, each bitline is connected to a sense amplifier. The set of sense amplifiers in a bank are called Row Buffer. A DRAM Cell is the basic element of a DRAM device since it is the actual storage element. Each Cell is built using an access transistor, controlled by the wordline, connected to a capacitor. When the worline is set high, the bitline is connected to the capacitor via the access transistor, allowing charge to flow into the sense amplifier.

\subsubsection{DRAM Operations and Commands}

Figure 2.4, extracted from (LEE et al., 2015), illustrates important phases that a DRAM's cell goes through during a request command. Initially, the cell is in the 
Figure 2.3: DRAM's Bank and Cell organization.

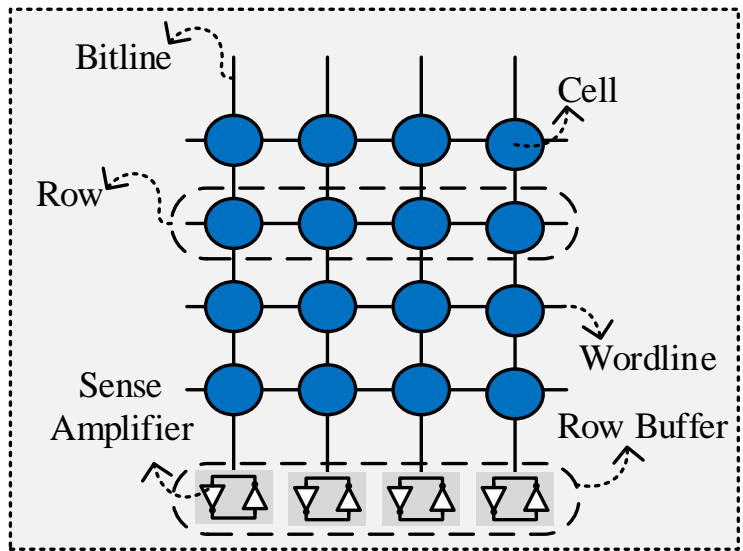

(a) DRAM Bank

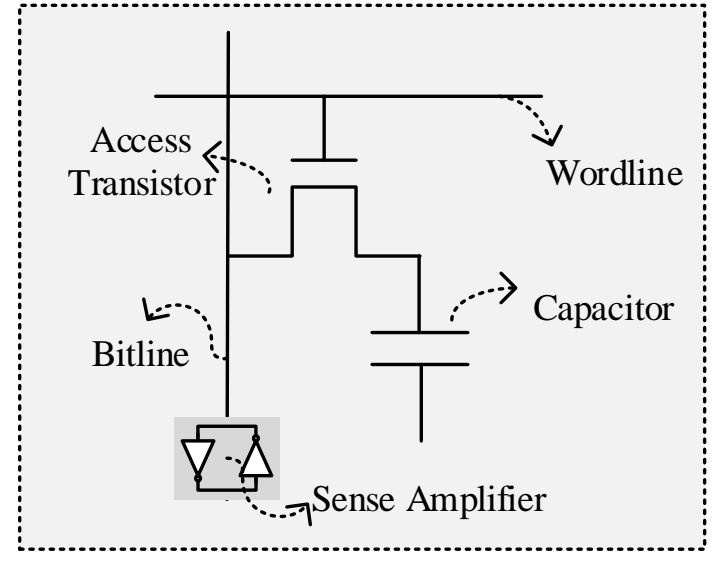

(b) DRAM Cell

Source: Provided by the author.

precharged state 1 , where the wordline is held in low, the bitline and the sense amplifier are maintained in an equilibrium of $1 / 2$ the $\mathrm{V}_{\mathrm{DD}}$ (maximum) voltage.

Activation. To access data from a particular row, the memory controller will send through the I/O Bus the row address related to that row and the ACTIVATE command. The row address is going to be decoded by the row decoder, and then the wordline related to the row is going to be set high (2). After that, all cells in the row are connected to their particular bitline, and each cell will lose/gain charge depending on its initial value. The charge will flow from the cell to the bitline if the cell is at a higher voltage state that the bitline, or from the bitline to the cell otherwise. This state is called chage-sharing. The sense amplifier will observe a positive or negative voltage difference on the bitline, and then fully restore the cell's charge based on that 3 .

Read/Write. When the sense-amplification process is completed, the sense amplifier will have stored the data related to the charge on the cell (either $0 \mathrm{~V}$ or $\mathrm{V}_{\mathrm{DD}}$ ), and also data will be restored into the cell 4 . The time the memory controller has to wait between

Figure 2.4: Overview of the DRAM's operation protocol.

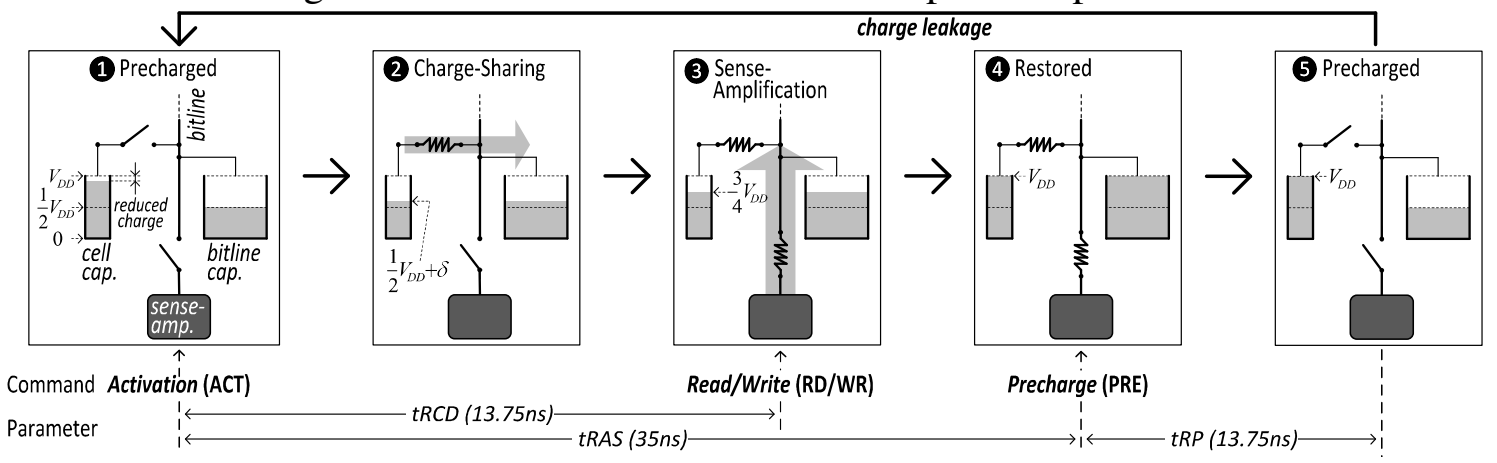

Source: (LEE et al., 2015). 
an ACTIVATE and a READ/WRITE command and data to be stable on the sense amplifier is called tRCD (row-to-column delay). After this time, the memory controller can send the column address related to the fraction of data it wants to access from the row buffer (a standard DRAM device has a row buffer of 4 Kbits or 8 Kbits). In traditional Double Data Rate (DDR) DRAM modules, new data will be available on the external data bus in both rising and falling edges of the clock. In general, the memory controller would try to maximize the number of consecutive access to the same row buffer (row buffer hit) since data can be obtained in a much smaller latency direct from the row buffer. This mechanism is called open-row policy. On the other hand, in a closed-row policy, a PRECHARGE command would be sent after all READ/WRITE commands, therefore cleaning the row buffer.

Precharge. When the memory controller issues a new request to a row different to the one already activated (row buffer miss), or in the case of a closed-page policy, the memory controller needs to emit a PRECHARGE command (5). During precharging, the wordline will be put in low, disconnecting the cell and the bitline. Also, the bitline needs to be set again to $1 / 2$ the $\mathrm{V}_{\mathrm{DD}}$. Therefore, the cell is ready to respond to a new request. The time between an ACTIVATE and a PRECHARGE command is called tRAS (row active time). This time parameter needs to be respected so data can be fully restored back to the cell. Also, after emitting a PRECHARGE command, the memory controller has to wait for tRP (row precharge time) between issuing a new command so that the precharging operation can be completed.

Refresh. Since the DRAM cell is a capacitor-based mechanism, it leaks charge over time. Therefore, all rows need to be accessed periodically (typically $64 \mathrm{~ms}$ ) to restore data back to the cell. This process is called REFRESH. During refreshing, a row is activated (opened), so that the sense amplifier can restore the cell's charge. The time parameter related to refresh is called tREFI (refresh interval).

\subsubsection{DDR Command Interface}

The memory controller issues request to the DDR device via the memory bus. When trafficking through the bus, DDR commands are encrypted into a set of five distinct signals: $C K E, C S, R A S, C A S$, and $W E$. The $C K E$ (clock enable) signas tells if the memory is activated (ready to be accessed) or in a low-power mode. The CS (chip select) signal enables a given rank of chips in the DIMM. The RAS (row address strobe) signal activates 
a row of cells, while the $C A S$ (column address strobe) signals is used to select a specific column within the row. These signals are combined with the WE (write enable) signal to generate $\mathrm{read} / \mathrm{write}$ requests.

\subsection{Hybrid Memory Cube}

Figure 2.5 depicts the internal organization of an HMC device. HMC is a 3D memory device that targets bandwidth-intensive applications. In the latest HMC specification (Hybrid Memory Cube Consortium, 2013), the device is composed of four high-speed serial links, a logic layer of 32 memory controllers (called Vault controllers), and four layers of DRAM memories connected via Through-Silicon Via (TSV) through the Vault controller. Each Vault controller can operate independently upon 16 memory banks, and also can execute some atomic arithmetic operations. A single HMC device can provide a total bandwidth up to $320 \mathrm{~GB} / \mathrm{s}$. With the logic and memory integration provided by HMC, the memory controller can be moved from today's internal CPU package to closer to the memory device, reducing overall system latency and improving performance for

Figure 2.5: Overview of the HMC organization. In this figure, a Partition is equivalent to a DRAM's rank.

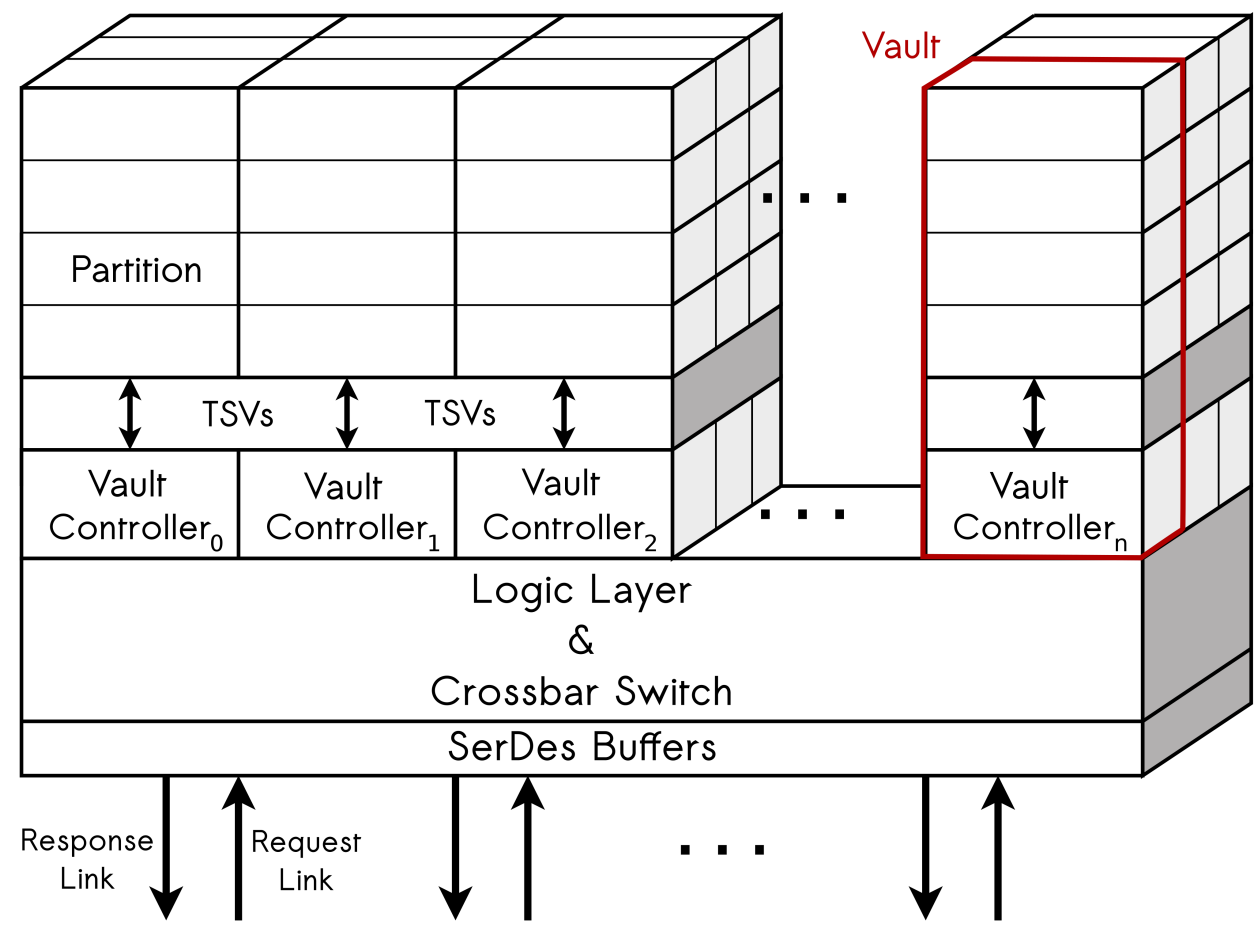

Source: Extracted from (ROSENFELD, 2014). 
Figure 2.6: Fields for request and response HMC packets.

\begin{tabular}{|c|c|c|c|c|c|c|}
\hline 6 & & & $24|23|$ & & 11 & 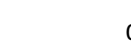 \\
\hline CUB[2:0] & $\operatorname{RES}[2: 0]$ & ADRS[33:0] & 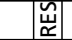 & TAG[10:0] & LNG[4:0] & CMD[6:0] \\
\hline
\end{tabular}

(a) Request header format.

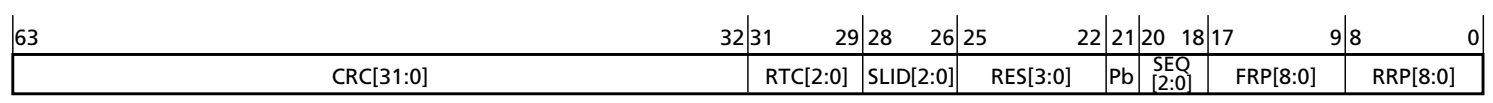

(b) Request tail format.

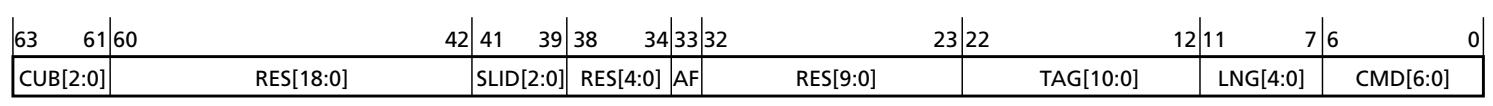

(c) Response header format.

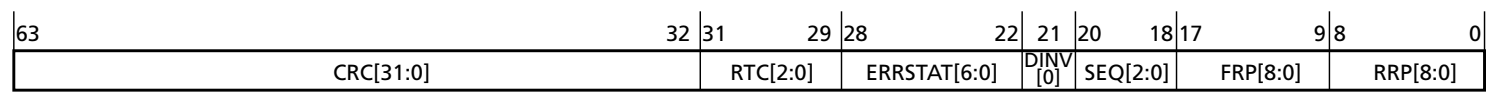

(d) Response tail format.

Source: HMC Specification 2.1. (Hybrid Memory Cube Consortium, 2013).

multi-core chips. Also, it is now possible to add computation resources alongside the logic layer of the HMC module. The HMC organization can be split into two major blocks. First, the Transceiver layer is responsible for managing request and response packets going between the HMC and the host device. Second, the Vault layer operates over these requests, accessing the DRAM layers to read/write data.

\subsubsection{Transceiver Layer}

HMC operates using a packet-based protocol. A packet, called FLIT, is 128 bits width and has at least a header and tail fields, each one being 64 bits width. Figure 2.6a illustrates the fields presented in the request header packet. It holds information regarding the requested HMC command (CMD), the number of FLITs in the request (LNG), a unique tag that identifies the request (TAG), the memory address (ADRS), and a module identifier (CUB). The CMD field is seven bits long and stores one opcode from the 54 possible HMC operations. Operations are categorized as READ, POSTED READ, WRITE, POSTED WRITE, and ATOMIC. READ and WRITE operations vary from 16 bytes to 256 bytes, in a 16 bytes step. All these operations return an acknowledge packet to the host. POSTED instructions have the same behavior than standard READ and WRITE operations but do not produce an acknowledge response to the host. The LNG field stores how many FLITs compose that specific request. The minimum number of FLITs per request is one, and the maximum is seventeen (for a 256 bytes request). The TAG field is 
responsible for identifying each packet. This value is used to ensure ordering between requests and responses throughout the system, and to identify a failing packet that may need to be re-sent. Finally, the CUB field stores an identifier for the particular memory cube, since several HMCs modules can be connected in a network to provide more memory capacity. Figure $2.6 \mathrm{~b}$ shows the fields presented in the request tail packet. Most of the information stored in the request tail is used to ensure correctness through the system. Since data traffic back and forth host and memory via high-speed serial lanes (up to $30 \mathrm{~Gb} / \mathrm{s}$ ), errors might happen. Therefore, the HMC protocol provides a mechanism to ensure packet integrity. The Return Retry Pointer (RRP), Forward Retry Pointer (FRP), and Sequence Number (SEQ) fields are used to manage retransmission of invalid packets. The Poison bit $(\mathrm{Pb})$ is set to one when the host wants to mark locations in the DRAM as having poisoned data. Source Link ID (SLID) indicates which one of the fours links the request came from. All response packets must be returned to the same source link. Return Token Count (RTC) indicates the number of free positions available in the request's input buffer. Finally, the Cycle Redundancy Check (CRC) field is used to verify the correctness of the packet.

Once the memory has finished processing the request packet, the Transceiver layer will generate a packet response to the host. Similar to the request packet, the response packet has a header and tail fields, plus any data payload related to the request. Figure 2.6c illustrates the response header. The CMD field specifies the request command that relates to the response. The LNG field tells how many FLITs compose the packet. The TAG field is the same identifier that came with the request packet. The Atomic Flag (AF) is set when overflow happens while executing arithmetic instructions. The SLID field indicates the link where the response should return. Finally, the CUB tells which HMC device the packet belongs. Figure 2.6d shows all fields in a response tail. The RRP, FRP, SEQ, RTC, and CRC fields serve the same purpose than the ones in the request tail. The Data Invalid (DINV) bit tells the validity of the packet payload. The Error Status (ERRSTAT) field is used to codify the type of error that might have happened during the Transceiver process, as warnings, DRAM errors, link errors, protocol errors, Vault errors, or fatal errors.

Figure 2.7 depicts the overall organization of the Transceiver module. The Requester and Responder sub-modules compose the Transceiver module. The former one is related to the host or any other HMC module that sends packets in the downstream flow, while the latter relates to the HMC module the sends packets in the upstream flow. 
Figure 2.7: Overview of the Transceiver module organization.

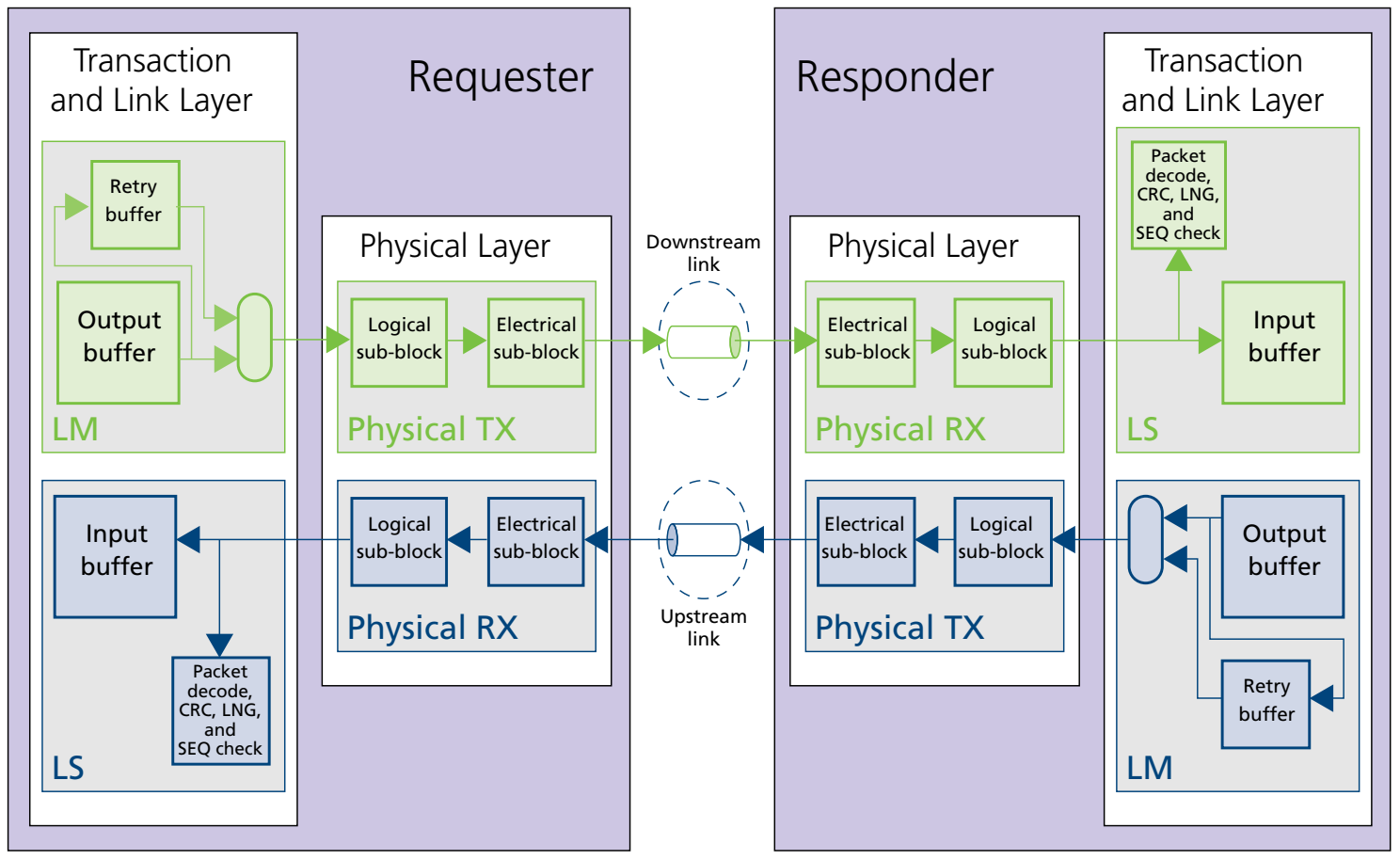

Source: HMC Specification 2.1. (Hybrid Memory Cube Consortium, 2013).

Also, both Requester and Responder sub-modules have a Transceiver and Link Layer and a Physical Layer. The Transceiver and Link Layer are responsible for handling the internal HMC communication protocol, encapsulating the requests and response packets and managing packet integrity. The Link Master (LM) is the module responsible for generating the packet, while the Link Slave (LS) receives and forwards the packet. Meanwhile, the Physical Layer serializes and de-serializes the packets produced by the Transceiver and Link Layer, and also manages transmissions across the high-speed serial links.

\subsubsection{Vault Layer}

The major modules within the Vault Layer are the Vault controller, the memory banks, some request/response buffers, and control logic. An HMC device is composed of 32 independent Vaults that can operate in parallel. Each Vault provides an aggregated bandwidth of up to $10 \mathrm{~GB} / \mathrm{s}$, which sums up to an internal aggregated bandwidth of $320 \mathrm{~GB} / \mathrm{s}$.

The Vault controller represents the same as the memory controller of traditional DRAM devices. It has five primary functionalities. First, to de-codify the packet coming from the Transceiver layer into DRAM's READ and WRITE commands. Second, to 
Figure 2.8: Representation of the low-interleave algorithm used as memory mapping scheme.

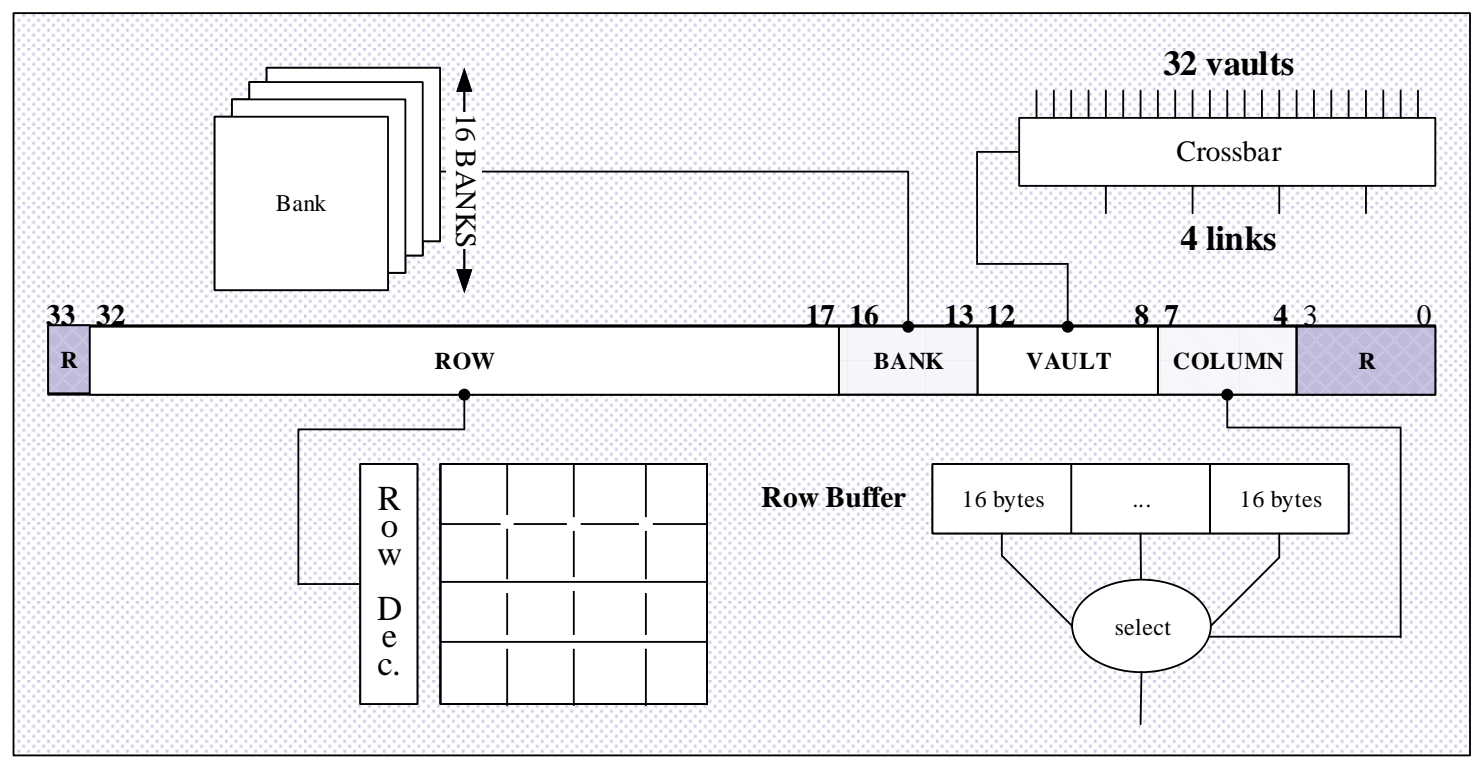

Source: Provided by the author.

schedule the memory requests in some order based on the row buffer policy. Third, to control the flow of data that navigates through the TSV to/from the DRAM banks. Fourth, to generate response packets to the host when appropriate. Finally, the Vault controller is responsible for preserving the order of all response packets, since even though it is possible to execute the memory requests out-of-order, they must be committed in-order to maintain data coherency. The TSV connects the memory banks to the Vault controller via a vertical bi-directional bus of 32 bytes. The bus is used to send the sequence of DDR commands generated by the Vault controller, the address related to the request, and data from READ and WRITE requests. Finally, the Vault controller has some logic units used to execute arithmetic operations generated by HMC atomic instructions.

The memory banks have the same internal organization as presented in Section 2.1. A single Vault controls 16 memory banks. For an 8 GBytes HMC module, a single bank stores a total of 16 MBytes of data. The row buffer size of the memory banks in an HMC device is much smaller than the one from standard DDR memories. While the latter ranges from $4 \mathrm{Kbits}$ from $8 \mathrm{Kbits}$, the former can be reconfigurated during initialization to be 32 bytes, 64 bytes, 128 bytes, or 256 bytes width. This difference in size is due to the fact HMC was originally designed for High Performance Computing (HPC) applications, where data locality is small at the row buffer level. In consequence, the row buffer policy employed natively by HMC is closed-row.

The row buffer size influences directly the interleave mechanism, i.e., how physi- 
cal addresses are mapped to the memory modules. The interleave used by HMC is called low-interleave and is illustrated in Figure 2.8. In this interleave algorithm, the four least significant bits of the memory address (34 bits in total) are ignored. The next up to four bits (the forth through up the seventh bits) address the number of 16 bytes segments within the row buffer. Then five bits are used by the crossbar switch between the Vault and Transceiver layers to address the target Vault. The next four bits address the banks, and finally, the remaining bits are used by the row address decoder within the bank. This algorithm favors subsequent requests since adjacent addresses are stored in sequential Vaults, and then in sequential banks within a Vault. Therefore, bank conflicts are avoided in requests for sequential addresses.

\subsection{The SystemC Programming Model}

Hardware Description Languages (HDLs), like Verilog and VHDL, have significantly increased the productivity level for hardware designers by creating a modeling environment that imposes a higher abstraction level than the previously schematic-only view. However, with the growth in complexity in modern hardware systems, the modeling platform employed would also need to be elevated its abstraction level. From modeling individual bits in HDL systems to simulating complex systems consisting of complex IP blocks, a vast number of processor cores, embedded software and other elements that might be included in today's System-on-Chips (SoCs).

Table 2.1 gives an overview of different programming models and whether or not they are appropriated for developing systems at distinct levels of abstractions. One can notice that high-level programming languages are a good modeling choice when only the primary system's behavior needs to be evaluated. It can be used to abstract the complexity involving the model's architecture, and provide an overview of how the mechanism will work conceptually. However, it does not provide detailed information regarding tailored timing parameters and the internal organization of the system. On the other hand, behavioral and Register Transfer Level (RTL) simulations can be obtained by employing HDLs, where each component of the system can be modeled taking into account their architecture, organization, and significant timing constraints. However, it can be a challenge to implement whole systems using these levels of abstraction, due to the scale and complexity of modern hardware systems.

SystemC (GHENASSIA et al., 2005) was created in 1999 by the Open SystemC 
Table 2.1: Programming languages categorized targeting distinct levels of abstraction.

\begin{tabular}{llll}
\hline Level of Abstraction & Verilog & VHDL & C/C++ \\
\hline System Level & No Suitable & Poor & Very Good \\
High Level (Behavioral) & Good & Very Good & Good \\
Medium Level (RTL) & Very Good & Very Good & Poor \\
Low Level (Gates) & Good & Poor & No Suitable
\end{tabular}

Source: Adapted from (JAYADEVAPPA; SHANKAR; MAHGOUB, 2004).

Initiative (OSCI, now Accelera) as a modeling platform that aims to elevate the abstraction level targeting to create a more flexible and productive hardware/software co-design environment. SystemC was built atop the $\mathrm{C}++$ programming language. Therefore, it takes advantages of the already presented object-oriented programming paradigm, the native data types, the inheritance and polymorphism capabilities, and all the software infrastructure already available for $\mathrm{C}++$. It also includes functionalities that are specific of hardware designs as concurrency, clocks, and bit-based data types.

Listing 2.1 shows a generic SystemC code with the major components of its programming model. It is composed of the module declaration, a set of input/output signals, a method, a list of sensitivity related to the processes, and potentially one or more submodules. A module is a container class that can be employed hierarchically to build each entity of the systems. A module typically has a set of input/output ports, processes, signals, and local variables. All modules must implement its constructor method, where submodules can be connected, and procedures are tight up with their respective sensitive list. A method defines one or more behaviors produced by the module. Each method is correlated to a sensitivity list of input ports. All methods within a module are executed concurrently and are scheduled to run when any port described in their sensitivity list changes.

Listing 2.1: A generic SystemC code snippet.

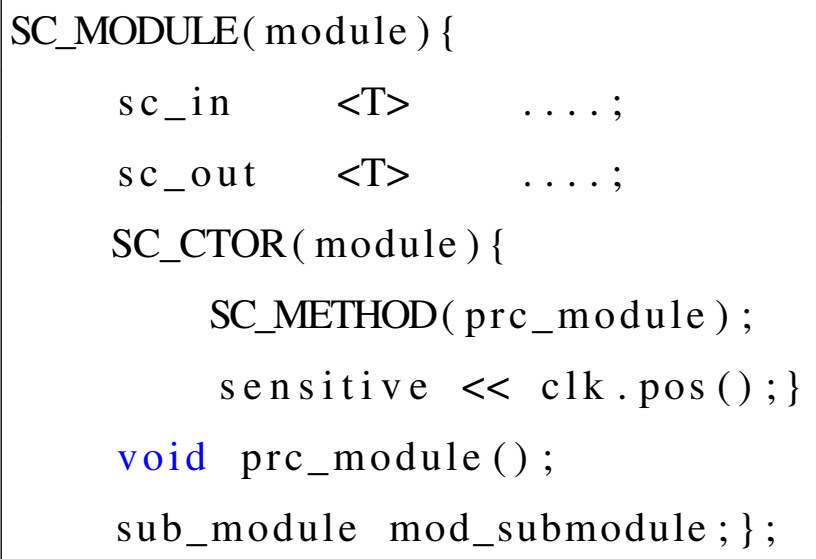




\section{RELATED WORK}

In this section, we present an overview of past works that aim to employ Processorin-Memory (PIM) architectures in diverse classes of application. Also, we depict a list of Hybrid Memory Cube (HMC) simulators available as open-source tools.

\subsection{Processing-in-Memory Architectures}

Several works have proposed to explore internal memory bandwidth and reduce data movement through the memory system by exploring new PIM architectures. During the 1990s, some authors provided mechanisms that integrated logic units inside DRAM chips (PATTERSON et al., 1997; ELLIOTT et al., 1999). However, due to manufacturing restrictions that DRAM devices face, this line of thought was abandoned until 3D-stacked technologies have became a reality. In this section, we list some past works that employ PIM designs targeting to accelerate distinct classes of applications.

First, the Tesseract mechanism (AHN et al., 2015a) aims to accelerate large-scale graph processing workloads by exploring the internal bandwidth of HMC. The device is composed of a set of 16 memory cubes. A simple ARM processor was inserted into each memory vault of all memory cubes (the whole mechanism has 512 ARM processors). Tesseract acts as a side accelerator to the host CPU, which is responsible for offloading the graph operations to the device. The authors also provided a programming interface that helps the accelerator's memory prefetches to explore the available memory bandwidth fully.

Second, in (HSIEH et al., 2016), the authors presented IMPICA, a PIM architecture to accelerate pointer-chasing in memory. The authors observed that structures like linked lists and B-trees have sparse memory access patterns, which harm systems' performance by increasing the number of cache misses. The mechanism integrates two different engines into the vault units inside the memory cube. One mechanism is responsible for translating virtual addresses to physical addresses efficiently, and the second one accesses the memory using the converted address taking advantage of memory parallelism.

Farmahini-Farahani et. al. (FARMAHINI-FARAHANI et al., 2014) proposed an architecture that consists of a Coarse-Grain Reconfigurable Array (CGRA) placed atop a stack of DRAM modules. The mechanism aims to accelerate scientific and embedded applications. The CGRA unit has 32 Functional Units (FUs) and receives 128 bits of data 
from the DRAM layers bellow. To execute an application, the user needs to create a data flow graph to be placed into the array. Then, data is read and write from memory via memory mapped I/Os.

In (AZARKHISH et al., 2016a), the authors proposed to include an ARM processor between the vault controllers and internal interconnection of an HMC device, targeting to reduce data movement and improve the performance of memory bounded applications. The authors also developed an interconnection network that connects the memory layers to the ARM processor. To execute an application, the processor offloads the required kernel to the processor placed inside the memory cube via a DMA engine. Virtual memory capabilities are provided to the application by a TLB unit inside the ARM core. (AZARKHISH et al., 2016a) was integrated with the gem5 simulator (BINKERT et al., 2011).

The Active Memory Cube (ACM) mechanism (NAIR et al., 2015) is a PIM architecture that can execute up to 1 Exaflops in $20 \mathrm{MW}$. Their device is composed of 32 processing lanes that are connected to HMC vault interconnection. (NAIR et al., 2015) has a set of vector register file that can efficiently explore the available memory parallelism. The authors provided a full suite of APIs and simulation tools to explore their design, including support for power and reliability calculation.

HMC Instruction Vector Extensions (HIVE) (ALVES et al., 2016) provides vector instruction capabilities to HMC modules. Each vector instruction operates over 8KB of data retrieved from the memory vaults. This architecture target applications whose behavior is similar to stream ones. HIVE is composed of 2048 functional units, capable of executing integer and floating point operations, and a register bank with eight registers of 8192 bits each.

In (XU; ZHANG; JAYASENA, 2015) it is proposed a PIM that targets Convolutional Neural Network (ConvNet). Their design is comprised of a network of four HMC modules and a host CPU. Each HMC is augmented with an accelerator layer of several Graphics Processing Units (GPUs) and CPU. To take advantage of their design, the authors have classified the layers of a ConvNet into two categories: explores data parallelism and explores model parallelism. When data parallelism is used, the input batch of images is partitioned equally between the PIM stacks. Thus each accelerator can execute independently of each other. On the other hand, when model parallelism is employed, the Neural Network (NN) itself is partitioned between the PIMs, allowing much larger ConvNets to execute. The authors do not provide information about the architecture of the employed 
GPU and CPU in the PIM layers and how the communication between modules is handled. Also, to evaluate their design, the authors make use of a machine learning-based simulator that only take into account the difference between bandwidth measures.

In (AZARKHISH et al., 2017), the authors presented Neurostream, a ConvNet architecture built as a network of $4 \mathrm{HMC}$ devices. In the logic layer of each memory cube, there is a Neurocluster device, which is composed of four RISC-V processors, 32 streaming co-processors built to accelerate multiply-and-accumulate operations. Their design was developed based on the observation that the most significative operation executed by today's ConvNet is the MAC one. The major drawback to their design is the fact that their accelerator layer is placed outside HMC vaults. Therefore, it is not possible to employ the maximum available bandwidth provided by the memory.

The authors of (OLIVEIRA et al., 2017) proposed NIM, a PIM-based approach that targets Spiking Neural Networks, another class of NNs. In this class of NNs, the primary challenge is that to evaluate each neuron it is necessary to evaluate complex mathematical equations under a strict time window. The authors have observed this application does not take advantage of the cache hierarchy due to the lack of significative data reuse between layers. Also, the time the application spent loading the parameters required to evaluate a single neuron represented was significantly notable when compared to the rest of the execution. In their design, the authors have elaborated tailored vector units placed inside the HMC vaults that were specialized to compute the required mathematical equations for two different neuron models.

Finally, in (SANTOS et al., 2017), the authors have observed that database operations have a similar behavior to streaming applications, i.e., there was not a significant amount of data reuse between different queries execution. Another observation they made was that, for queries that have a composed condition statement (SELECT * FROM table WHERE [a AND b AND c ...]), data requests could be reconfigurable based on the result of the previous conditional statement. With these two notes in mind, the authors built a simple reconfigurable PIM mechanism to accelerate database operation, improving energy efficiency by reducing the total amount of data that needs to travel through the whole memory system. 


\subsection{Processing-in-Memory Simulators}

Since the release of the first 3D-stacked memory, several attempts to build a concise simulator have been made by different researcher groups. However, it is not an easy task to replicate those devices functionalities because their internals are not available as open source information. Besides that, most of the simulators presented in this section have been implemented using sequential high-level programming languages. This methodology faces some issues since memory modules have a highly-parallel behavior. For example, any cycle-accurate simulator implemented using a non-parallel language would need to check at every single clock single the current state of all modules in the simulation, causing long simulation times.

From all currently available HMC simulators, HMC-Sim (LEIDEL; CHEN, 2016) is the most similar to our framework. This simulator was developed using the $\mathrm{C}++$ programming language and provides a cycle-accurate memory simulation. Also, the simulator provides a simple data-structure based interface. Thus one could extend the already presented HMC instructions. Even though their approach can assist one to investigate the PIM capabilities of an HMC device, it has some limitations. First, to implement custom instructions, the authors have taken advantage of all opcodes that are not being used by the current HMC specification. However, this methodology adds a scalability problem to their interface due to two factors. First, only seventy (the number of currently free opcodes) new instructions can be created by the user, and second, as it had happened from HMC specification 1.0 to 2.0 (Hybrid Memory Cube Consortium, 2013), new native instructions are introduced by the HMC Consortium, making use of reserved opcodes. Finally, since shared library objects were used in their framework to provided a friendly interface to include the new HMC instructions, only Unix users can take advantage of the HMC-Sim framework. One significant difference from (LEIDEL; CHEN, 2016) and the presented work is that our PIM interface allows the user to include new architectures into HMC logic layer, rather than extending the already included instructions.

CasHMC (JEON; CHUNG, 2016) is a C++ HMC simulator that provides full HMC capabilities. It is an offline simulator that uses an external memory trace as its input generated by any processor simulator. This simulator aims to implement most of the HMC resources, as packet error detection, link flow control, and HMC instructions, while providing to the user some output files as performance summary, trace logs, and simulation graphs. This approach can lead to longer simulation times since the simulator 
needs to write information to four different files in each clock since. Besides that, the simulation accuracy can be profoundly affected by the fact that the simulation does not run alongside the program execution. Finally, CasHMC does not provide any PIM extension.

The Smart Memory Cube (SMC) Simulation Environment (AZARKHISH et al., 2016a) is a complete set of applications built inside the gem5 framework targeting PIM architectures. The simulator makes use of the already present memory modules, interconnection networks, and CPU implementation to create the HMC device. Also, it provides a software stack, including drivers and code annotation, to forward instructions to the processor core inside the memory device. Even though SMC-Sim is a complete and welldeveloped set of tools that can help the user to investigate the advantages of 3D-stacked memories, it is possible to point out some implementation choices that may limit performance exploration. First, the PIM layer is located between the memory controllers and the interconnection layer. Therefore it is not possible to extract all the bandwidth provided by the memory because a significant portion of bandwidth contention exists at this area. Second, although Azarkhish et al. (2016) have claimed to have validated their design latency and performance parameters using a complete Register Transfer Level (RTL) design, the memory implementation inside the gem5 simulator is based on correlations between Double Data Rate (DDR) and HMC architectures. This approach is valid in most parts, but the HMC architecture presents some particular characteristics that are not present in current DDR controllers, like Through-Silicon Via (TSV) access control. Finally, to obtain significant performance from their simulator, the authors have employed some structural modifications to the current HMC implementation, for example, changing the maximum request size from $256 \mathrm{~B}$ to $512 \mathrm{~B}$, increasing the number of interconnection from 4 to 8 , while duplicating its data width from $128 \mathrm{~b}$ to $256 \mathrm{~b}$. Some previous studies, as (ROSENFELD, 2014), have shown that it is possible to extract close to the theoretical HMC bandwidth using the already presented design modules.

Finally, many in-house simulators have been employed during the past few years to obtain information regards PIM advantages. To illustrate, the author of (ALVES et al., 2016) have used a functional HMC simulator to implement different accelerators that target vector operations, database, and deep learning applications. Stelle et. al. (2014) use a Structural Simulation Toolkit (SST) simulator to investigate how data and computational locality impact PIM design. ZHang et. al. (2014) implement an analytic simulator aiming to understand how High Performance Computing (HPC) applications can benefit from 3D-stacked memories. Sura et. al. (2015) present a set of methodologies that can 
Table 3.1: Comparison between available PIM simulators. "?" indicates the information could not be obtained.

\begin{tabular}{|c|c|c|c|c|c|c|c|c|}
\hline Simulator & $\begin{array}{l}\text { Simulation } \\
\text { Accuracy }\end{array}$ & $\begin{array}{l}\text { Native PIM } \\
\text { Capabilities }\end{array}$ & $\begin{array}{l}\text { Novel PIM } \\
\text { Capabilities }\end{array}$ & $\begin{array}{l}\text { Peak } \\
\text { Bandwidth }\end{array}$ & $\begin{array}{l}\text { Deal with } \\
\text { Parallelism }\end{array}$ & $\begin{array}{l}\text { Input } \\
\text { Type }\end{array}$ & $\begin{array}{l}\text { Parame- } \\
\text { terizable }\end{array}$ & $\begin{array}{l}\text { Simulation } \\
\text { Time }\end{array}$ \\
\hline HMC-SIM & $\begin{array}{l}\text { Cycle- } \\
\text { Accurate }\end{array}$ & Yes & $\begin{array}{l}\text { Yes - } \\
\text { Limited }\end{array}$ & $320 \mathrm{~GB} / \mathrm{s}$ & $\begin{array}{l}\text { Event } \\
\text { queues }\end{array}$ & $\begin{array}{l}\text { Offline } \\
\text { Trace }\end{array}$ & No & $?$ \\
\hline Cas-HMC & $\begin{array}{l}\text { Cycle- } \\
\text { Accurate }\end{array}$ & Yes & No & $?$ & $\begin{array}{l}\text { Event } \\
\text { queues }\end{array}$ & $\begin{array}{l}\text { Offline } \\
\text { Trace }\end{array}$ & No & $28.7 \mathrm{~s}$ \\
\hline SMC & $\begin{array}{l}\text { Cycle- } \\
\text { Accurate }\end{array}$ & Yes & Yes & $205 \mathrm{~GB} / \mathrm{s}$ & $\begin{array}{l}\text { Event } \\
\text { queues }\end{array}$ & $\begin{array}{l}\text { Online } \\
\text { Trace }\end{array}$ & Yes & $4.5 \mathrm{~s}$ \\
\hline
\end{tabular}

Source: Provided by the author.

help reducing access latency of 3D memories. To evaluate their proposal, the authors implemented a timing-accurate simulator.

Today's PIM simulators can be categorized into three broad groups: functional, cycle-accurate, and analytical models. Analytical models provide faster simulations but may not take into account important design metrics. Functional models execute only the behavior of the target architecture, instead of implementing and simulating the internals of the architecture itself. Cycle-accurate models are the most precise but can lead to slow simulations. Our simulator fits the latter category, but since we have used a high-level hardware description language to build our design, the simulation time is significantly lower than previous implementations. Also, our proposed framework has been constructed following the most current HMC specifications.

To summarize, Table 3.1 list the most important open-source HMC simulators available currently. Each simulator is categorized by their simulation' accuracy, if it implements the native HMC 's PIM instructions, if it provides any means to include novel PIM architectures into the simulator, the peak bandwidth obtained by the simulator, its type of input file, whether or not the simulator is parameterizable, and the time it took to simulate $1 \mathrm{M}$ read instructions. As one can notice, all listed simulators claim to be cycle-accurate, implement the native HMC atomic instructions, and deal with parallelism using event queues. While HMC-SIM provides the user the ability to include novel PIM instructions by extending the already present HMC atomic instructions, SMC allows the user to insert a whole architecture within the HMC logic layer. However, as aforementioned, SMC cannot achieve HMC peak bandwidth. SMC is the only online simulator, meaning it can run alongside a CPU simulation. It is the only parameterizable simulator, while also being the fastest. 


\section{SIMULATOR IMPLEMENTATION}

This section describes in details our simulator's architecture and discusses some design choices we have made during the implementation process. To implement the simulator, we have carefully studied the current Hybrid Memory Cube (HMC) specification (Hybrid Memory Cube Consortium, 2013) and then elaborated each component described in the documentation.

The presented simulator, called Cycle Accurate Parallel PIM Simulator (CLAPPS), is divided into three major blocks: Vault, Transceiver, and Traffic Monitor. The first one is responsible for receiving a memory request, translating the request into memory commands, scheduling and executing the generated memory operations, and generating the appropriate response packet for either a READ, WRITE, native Processor-in-Memory (PIM), or custom PIM request. The second one is responsible for encapsulating requests coming from the host, sending and controlling the request flow from the host to the Vault and vice-versa. Finally, the latter one is a pure $\mathrm{C}++$ model that is responsible for tracking the request/response flow to produce statistics at the end of the simulation.

Figure 4.1 provides a simplified view of CLAPPS's UML packet diagram. Due to the complexity of the system, we broke-down the major modules into sub-modules and give a detailed description of their functionalities and interconnections.

Figure 4.1: Simplified packet diagram of the proposed simulator.

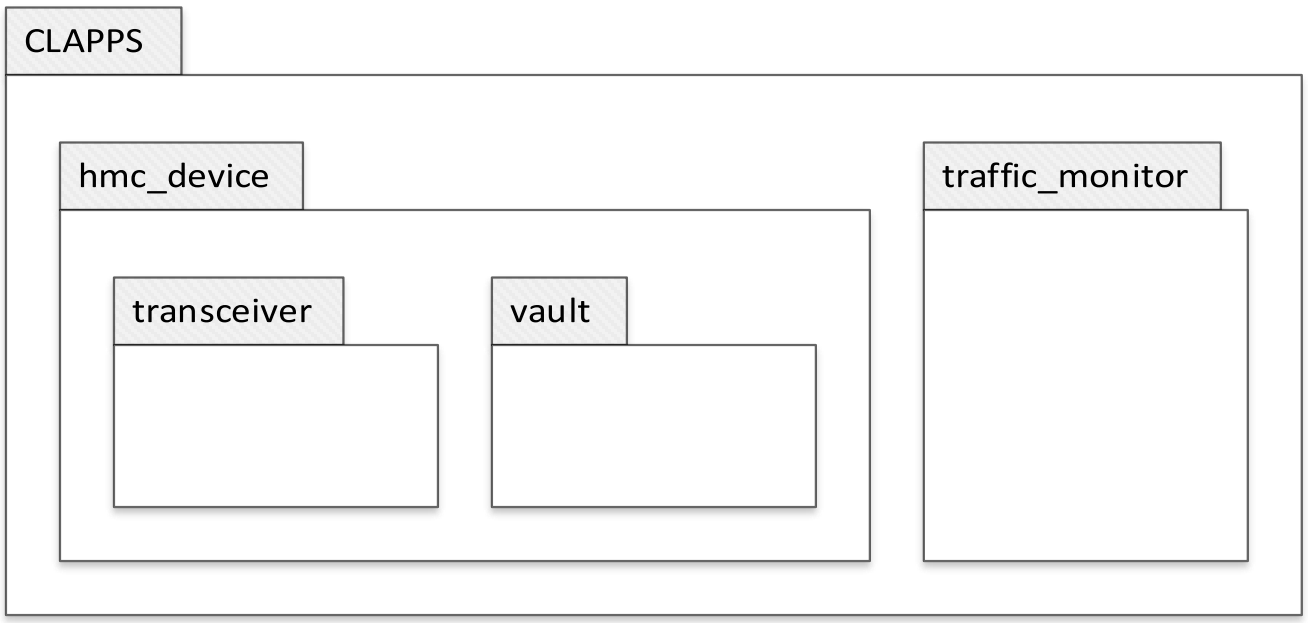

Source: Provided by the author. 


\subsection{Transceiver}

Figure 4.2 shows the packet diagram of the Transceiver module. As aforementioned in Section 2.2, the Transceiver is divided into two big blocks: the Requester and the Responser modules. First, we described the Requester block, its internal modules and operation, and then the Responser block.

\subsubsection{Requester}

The Requester module is composed of the transaction and link layer and physical layer sub-modules. The former one is responsible for implementing the HMC communication protocol. It provides the definition of FLITs and also ensures that order between

Figure 4.2: Simplified packet diagram of the Transceiver Layer.

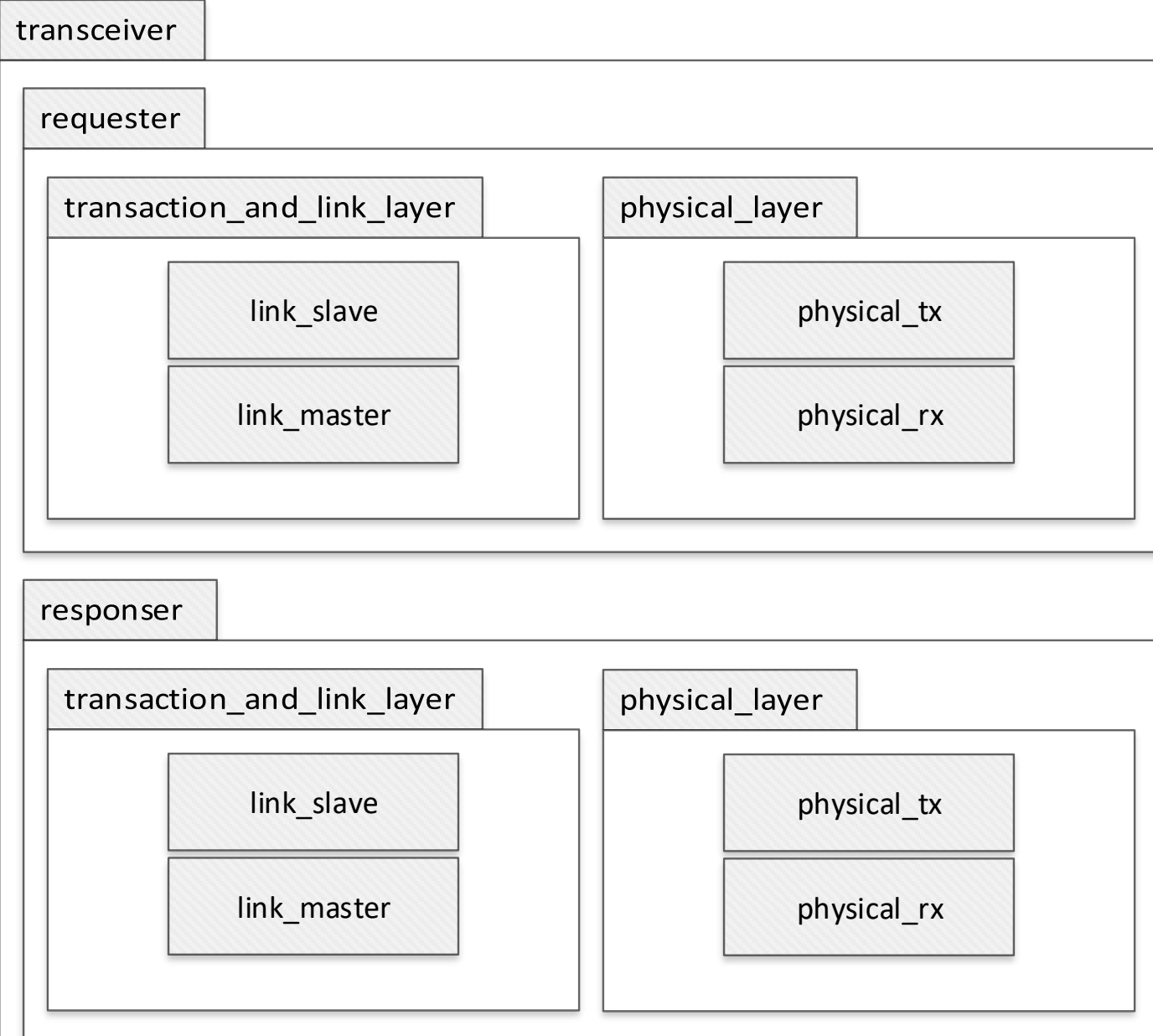

Source: Provided by the author. 
Figure 4.3: Block diagram of the packet generator module.

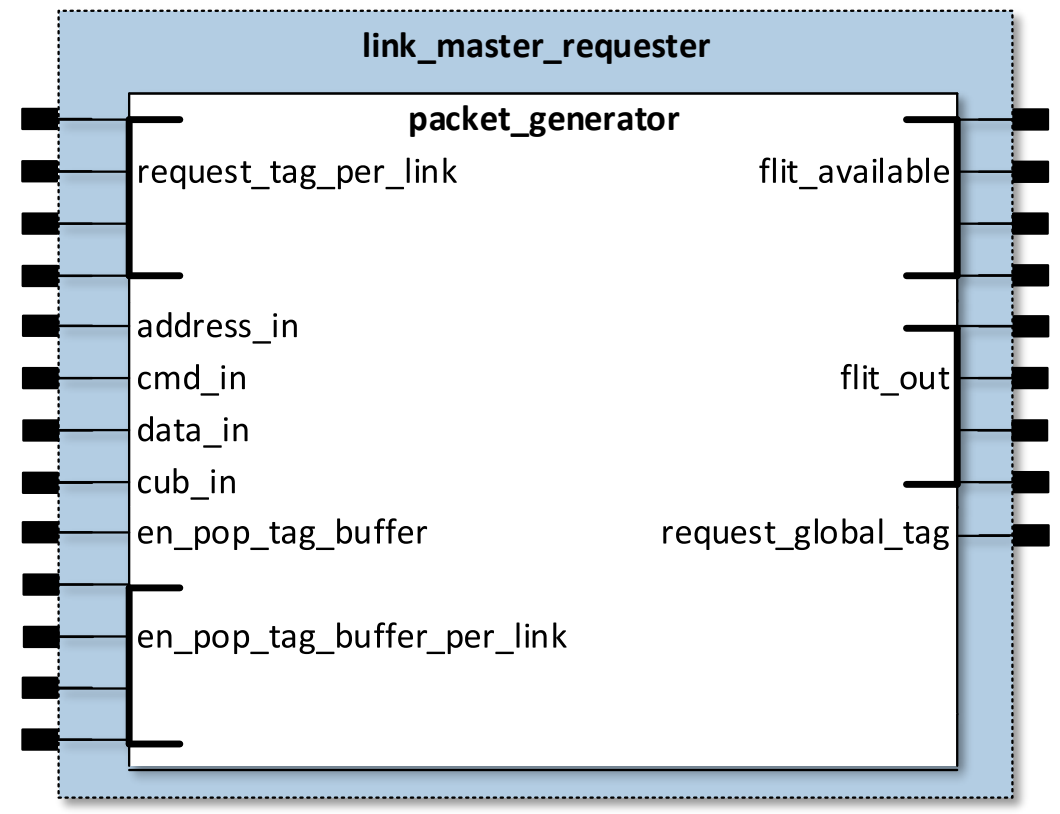

Source: Provided by the author.

requests is maintained. The latter one controls the high-speed serial links by serializing and de-serializing request and response FLITs. The transaction and link layer is once again divided into two sub-modules: link master and link slave. The link master encapsulates the request command that comes from the host, stores all generated FLITs at a local queue related to each link and also saves the generated tag identifier of the current request. Meanwhile, the link slave receives the response FLITs that come from the Vault, and stores them into the target link response buffer. Then, it reads all tag values that come from all link response buffers and based on the identifier stored at the link master queue it allows the appropriate link response buffer to send its FLITs back to the host. The physical layer is also split into two other sub-modules: physical tx and physical $r x$. While the physical tx sub-module reads FLITs stored in each link queue and serializes them into chunks of 16 bits, the physical rx sub-module reads chunks of 16 bits of data that come from the Responser module and reconstructs them into whole 128-bit width FLITs.

The primary module inside the link master sub-layer is the packet generator. Figure 4.3 illustrates the packet generator internal organization. The module works as follows. First, when a change happens in either the address, cmd, data, or cub_id input, the prc_flit_generator() method is launched. This method will first create the header and tail portions of the FLIT related to the new request using the create_header() and create_tail() functions. In the former one, the decode_cmd() function is called, so the number of FLITs necessary by this request can be generated appropriately. After that, the generate_tag() 
Figure 4.4: Block diagram of the link slave module.

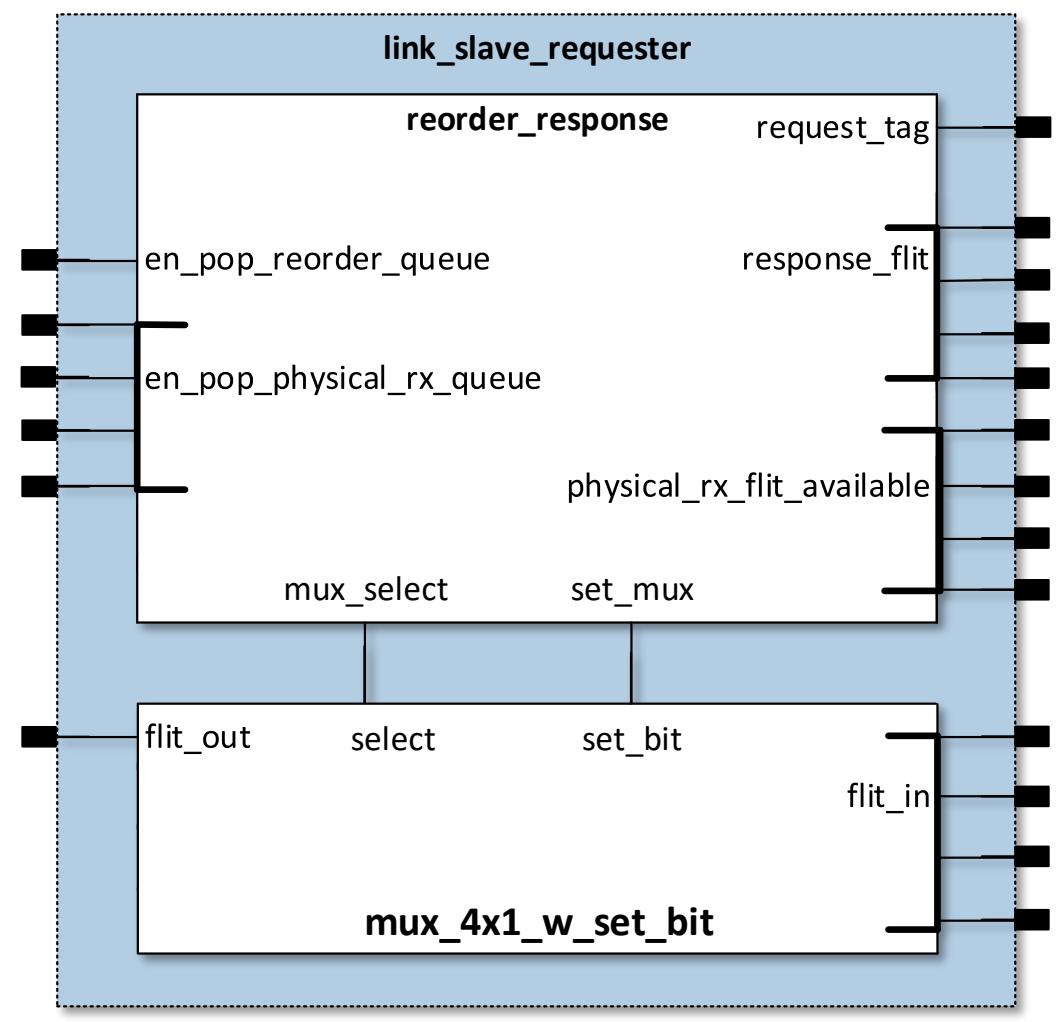

Source: Provided by the author.

function creates a unique tag identifier to the request. This tag is then stored into the global tag buffer. When creating the tail part of the packet, the link_to_send() function is called to indicate the path the request should follow through one of the four links. This decision is made by selecting the destination link in a round-robin fashion. Finally, in the case of a WRITE request, the appropriate chunks of input data are inserted into the FLITs, and then the packet ${ }^{1}$ can be stored in the local buffer related to the chosen transition link. The prc_send_fit_to_physical_tx() method runs at all positive edges of the clock, and is responsible for reading the link's request buffer, and writing the request at the top of the buffer in the flit_out port, and also rising the flit_available signal. To conclude, the prc_check_tag_ordering() reads the global tag buffer and writes its value at the request_global_tag signal during all positive clock edges.

In the other hand, as illustrated by Figure 4.4, the link slave module has two submodules, the reorder_response, and the mux_4x1_w_set_bit. The first one is an Finite State Machine (FSM) that keeps track of the order which the link master module generated packets, and based on that, selects the appropriate response packet to send back to the host. To do so, it reads the tag at the top of the global tag buffer at the link master module and

\footnotetext{
${ }^{1}$ We assume during the rest of the text that a packet is equal to the set of FLITs generated.
} 
the available FLITs that come from the downstream traffic. If any of the four links have a response packet with a tag field matching the one at the global tag buffer, the multiplexer is set to this link, and the path between link and host is locked until all FLITs from the response packet have been sent. When this process is concluded, the reorder_request sends a pop signal to the link master module so that the next global tag can be output.

Figure 4.5 depicts the physical layer module. This module is built using two submodules, a serializer (physical tx) and a de-serializer module (physical $r x$ ). Each link has its own set of serializer/de-serializer modules. The serializer (Figure 4.5a) splits the 128bit FLIT request into eight chunks of 16 bits and then transmits the fragmented pieces to the high-speed serial lanes. All four serializers can work in parallel, i.e., sending different requests at the same time, but only one request can navigate through a link per time. Therefore, one link must conclude to service (transmit) all FLITs of a request before switching to another request. On the other hand, the de-serializer (Figure 4.5b) receives chunks of 16 bits of data and reconstructs the FLIT so that it can be sent to the transaction and link layer.

Figure 4.5: Block diagram of the physical layer.

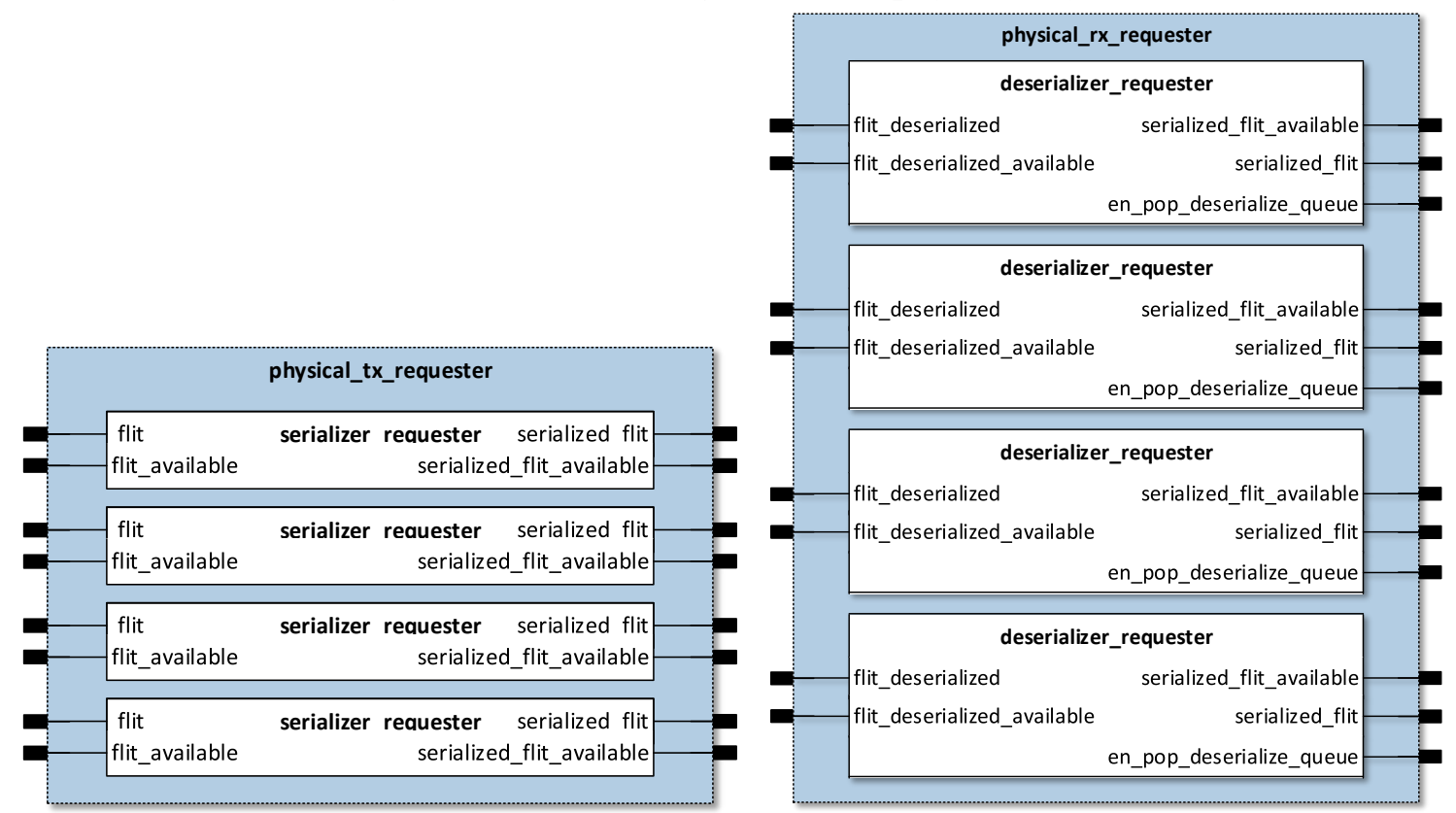

(a) Physical tx module. (b) Physical rx module.

Source: Provided by the author. 


\subsubsection{Responser}

Similarly to the Requester module, the Responser module has two internal submodules, the physical and transaction and link layers. The physical layer has the same functionality as the Requester's one. It serializes FLITs that come from the Vaults and de-serialize the ones received from the Requester.

Figure 4.6 illustrates the link slave organization. The module is composed of a four by four crossbar network, four one by eight de-multiplexers, and the link control. The interconnection between the four serial links and the 32 Vaults is a critical performance bottleneck for an HMC device. Potentially, all four links will be sending data for all the 32 Vaults, and vice-versa, creating a producer-consumer scenario. Ideally, this interconnection would have low-latency to transmit packets, would be no-blocking, and also would be scalable in size. However, it is not possible to always address these three characteristics at the same time. For example, a non-blocking interconnection network, as a crossbar network, does not scale well in size ${ }^{2}$. Meanwhile, a smaller and scalable interconnection network, as a butterfly networks, will potentially generate conflicts between accesses. Azarkhish et. al. (2014) have addressed this problem by proposing to employ a Network-on-Chip (NoC) as the interconnection network between Vaults and Links. The proposed NoC was based on the AMBA AXI-4.0 standard (AMBA, 2003). Even though this solution provided a non-blocking and scalable interface, it added extra latency to the Transceiver mechanism.

To cope with this problem, we have decided to implement a two-level interconnection network. The first level connects the four links to four quadrants using the $4 \times 4$ crossbar switch. As specified by (Hybrid Memory Cube Consortium, 2013), a quadrant is a group of eight Vaults. Then, each quadrant is connected to the Vaults using either an $8 \times 1$ multiplexer (in the downstream flow) or a 1x8 de-multiplexer (in the upstream flow). Consequentially, we can archive a low-latency and non-blocking communication at the first level, and a low-latency and blocking communication at the second level. Regarding scalability, the crossbar switch now grows with the number of quadrants, in contrast to a fully-connected 4x32 crossbar that would grow in terms of the number of Vaults.

The link control is responsible for managing the interconnection network and works as follows. At the link slave, when the physical layer sends a new FLIT, the link control reads the packet's header to obtain the address, and the number o FLITs related to

\footnotetext{
${ }^{2}$ In fact, a crossbar network with $\mathrm{N}$ inputs and $\mathrm{M}$ outputs will require $O(N M)$ switches.
} 
Figure 4.6: Block diagram of the link slave module

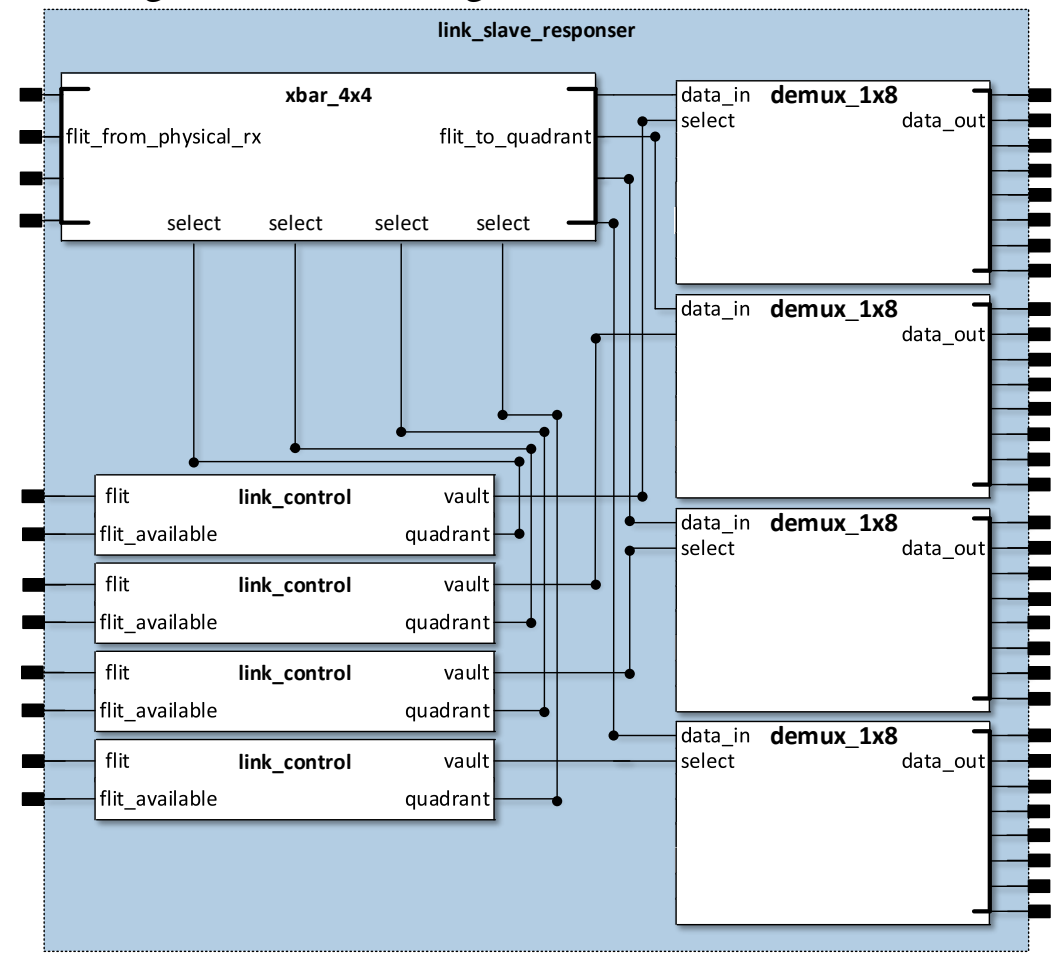

Source: Provided by the author.

the request. The address is important because it tells the destination Vault, based on the interleaving being employed. The number of FLITs is required because the link control must ensure that all FLITs from the request are sent prior servicing a new request. Then, it sets the crossbar network and the de-multiplexer based on the obtained Vault address. Besides that, it stores in the tag_per_link queue the tag related to the request.

At the link master (downstream flow), the link control reads the FLITs available from the Vaults, checking if there is a response tag that matches the tag at the top of the tag_per_link queue, therefore ensuring ordering between response packets within a quadrant. If the required response packet is ready to be sent, the link control sets the multiplexer and the crossbar to close the path between the Vault and the physical layer. At the end of the process, the link control sends a pop signal to remove the current tag at the top of the tag_per_link queue, allowing the next request can be serviced.

\subsection{Vault}

Figure 4.7 shows the UML packet diagram of the Vault module. The Vault module is similar in functionality to Double Data Rate (DDR) Dynamic Random Access Memory (DRAM)'s memory controller. However, due to intrinsic characteristics of 3D- 
Figure 4.7: Simplified packet diagram of the Vault Layer.

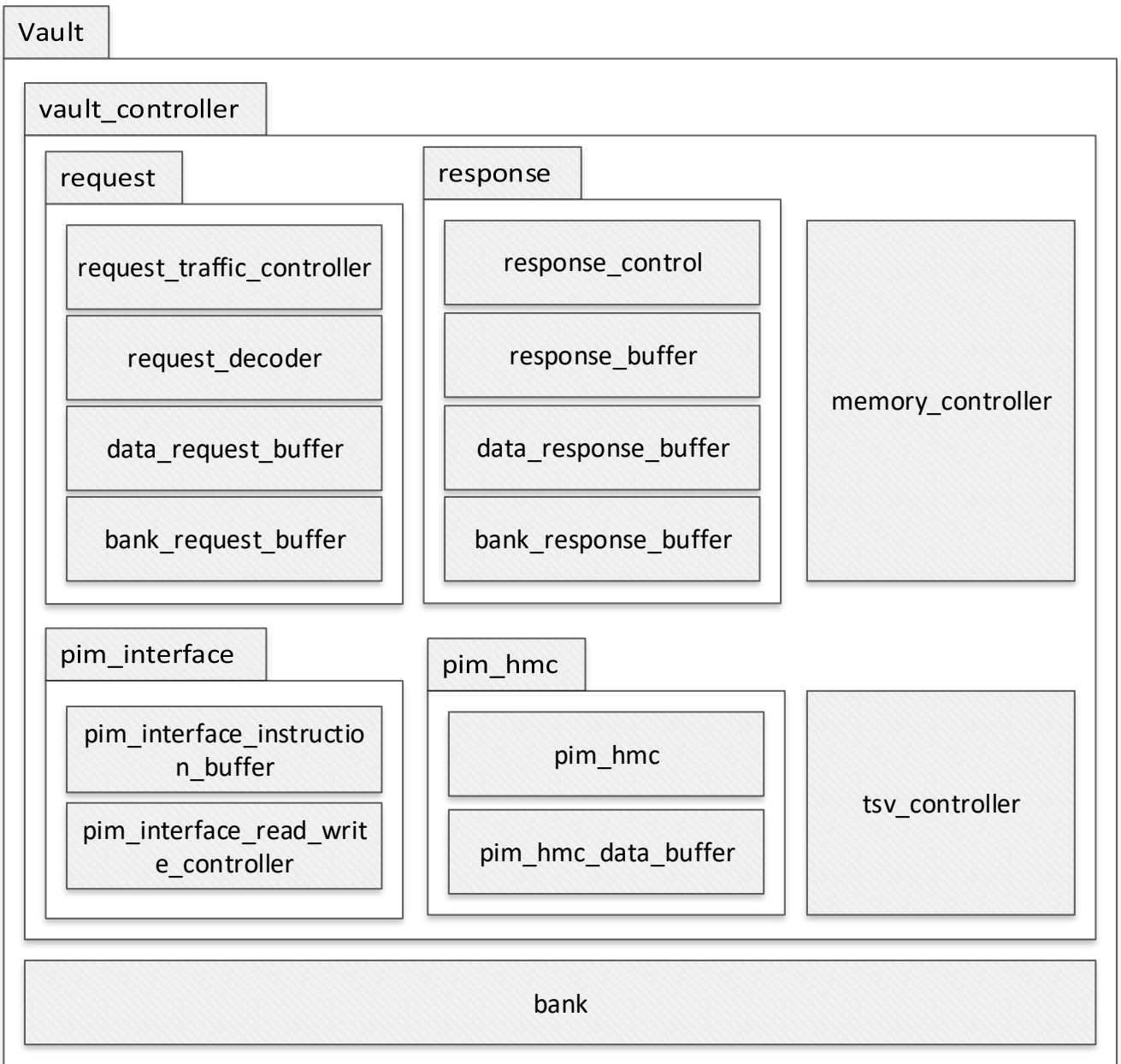

Source: Provided by the author.

stacked memories and also to respect the HMC protocol, diverse modifications to a simple DRAM's memory controller must be employed. The Vault is divided into Vault Controller and Bank. The first one has the following sub-modules: request, response, memory controller, Through-Silicon Via (TSV) controller, PIM HMC, and PIM interface. The PIM interface is the principal contribution of this work. The last one implements the functionalities of a DRAM bank.

In the following sections we discuss all the sub-modules present in the Vault. In contrast to the Transceiver module, the HMC Consortium (Hybrid Memory Cube Consortium, 2013) does not give information related to the Vault internal organization, TSV width and operational frequency, the number of banks that can be opened per time, and native PIM instructions' implementation. To overcome this issue, we have gathered information related to the Vault implementation from previously published works, in special from (AZARKHISH et al., 2016b), (MATHEW et al., 2017), (ROSENFELD, 2014), and (LEE et al., 2016). From (AZARKHISH et al., 2016b), we could obtain a general view 
Figure 4.8: Block diagram of the Vault Request module.

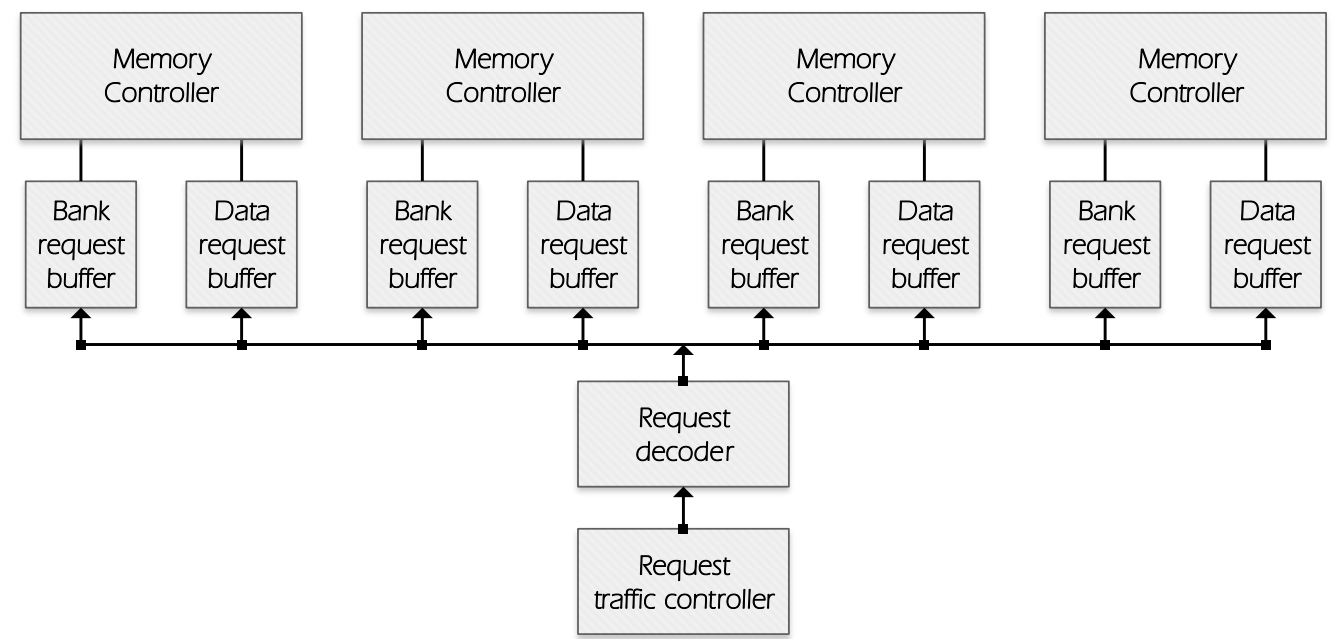

Source: Provided by the author.

of the Vault organization, and how the elements of the module correlate to traditional memory controllers. From (MATHEW et al., 2017), we gather the timing parameters and operational frequency for the DDR memories, and also the Vault operational frequency. From (ROSENFELD, 2014), we obtained information about the expected memory bandwidth for diverse set of Vault configurations. Finally, from (LEE et al., 2016), we extracted information about how the TSV is expected to work.

\subsubsection{Request}

Figure 4.8 shows the block diagram of the Vault Request module. This module is composed of the request_traffic_controller, request_decoder, bank_request_buffer, and data_request_buffer sub-modules. The request_traffic_controller is responsible for managing the stream of FLITs coming from the host and from the PIM Interface (we give more information about the PIM Interface in Section 4.2.5). Inside the module, an arbiter decides which request's flow can send its FLITs to the Vault in a round-robin fashion. Therefore, if only requests from the host-side or only from the PIM Interface-side of the flow are coming to the Vault, the request_traffic_controller just forwards the FLITs to the Vault without checking the arbiter mechanism. On the other hand, if requests are coming from both host and PIM Interface, the request_traffic_controller checks the round-robing counter and forwards the request of the flow that has priority. At the end of the traffic, the module increments the round-robin counter and serves the next requests.

The request_decoder module is the primary component of the Vault's request 
Table 4.1: Description of th request_decoder module, its input/output ports, methods, and functions.

\begin{tabular}{|c|c|c|c|c|}
\hline Inputs & & Outputs & Methods & Functions \\
\hline $\begin{array}{l}\text { start_decoding } \\
\text { request }\end{array}$ & $\begin{array}{c}\text { data_from_packet } \\
\text { tag } \\
\text { link } \\
\text { cub_id } \\
\text { response_lng } \\
\text { cmd } \\
\text { pim_instruction } \\
\text { operation } \\
\text { operation } \\
\text { response_dst }\end{array}$ & $\begin{array}{c}\text { en_bank_data_buffer } \\
\text { en_bank_response_buffer } \\
\text { en_response_buffer } \\
\text { packet_to_pim_interface } \\
\text { en_pim_instruction_buffer } \\
\text { address_offset } \\
\text { alu_operation } \\
\text { bank_id } \\
\text { address } \\
\text { en_bank_operation_buffer }\end{array}$ & prc_decode & $\begin{array}{c}\text { header_decoder } \\
\text { tail_decoder } \\
\text { decode_address } \\
\text { decode_cmd } \\
\text { bank_policy } \\
\text { operation_size }\end{array}$ \\
\hline
\end{tabular}

Source: Provided by the author.

block. It reads the upcoming FLITs and translates the request into READ/WRITE commands, which the memory controller can understand. The module also schedules the request to the appropriate memory controller, aiming to explore the maximum bank-level parallelism by avoiding bank conflicts. Also, it stores upcoming data from the request into the bank's data request buffer. Finally, it sends important package-information that comes with the request to the response module.

Table 4.1 specifies the internal organization of the request_decoder module, its set of input and output ports, methods, and functions. The module works as follows. During all rising edges of the clock, the prc_decode() method checks if the start_decoding signal was raised. When the signal is set, the module starts decoding the upcoming flow of FLITs. To keep track of the number of FLITs related to the request, the module has an internal counter, which is initialized with zero during reset. If the counter is equal to zero during decoding, it means that the module is dealing with the first FLIT of the request. Then, the header_decoder() function is called to split the header's fields into command opcode, tag, the number of FLITs within the request, address, and cube. With the command opcode, the module can call the decode_cmd() function to translate the HMC instruction into simple READ/WRITE/ATOMIC commands, obtain the size of the operation, and the number of response FLITs that the response module will generate when the request process concludes. Also, the header_decode() calls the decode_address() function to obtain the bank address targeted by the current request, the address within the bank, and starting position data should be read/write in the row buffer. 
When the header_decode() function returns, the bank_policy() function is called to decide which memory controller should manage the current request. This decision is made based on the target bank from the request, and the banks that are currently being serviced by the memory controllers. If any memory controller is servicing the same bank targeted by the current request, the request is sent to this memory controller. Otherwise, the request is sent to the next memory controller in a round-robin fashion. To simplify our simulator's design, we decided to correlate the number of memory controllers that can be operating in parallel with the tFAW DRAM timing parameters. This parameter specifies the number of rows that can be kept open at the same time, without overheating the chip. Since the HMC row policy is defined as close-row, there is no row buffer parallelism to explore. Therefore, it is possible to implement several memory controllers working in different banks in parallel, instead of a single memory controller managing several banks without losing performance because the memory controllers do not need to share row-level information. In today's DDRx modules, the tFAW is up to four. Therefore we implemented four memory controllers into the Vault. However, one could potentially keep all 16-row buffers (there are sixteen banks per Vault) open at the same time to explore the available bank level parallelism dramatically ${ }^{3}$.

Following the request decoding process, after decoding the header and deciding the destination memory controller, it is required to determine if the upper part of the current FLIT represents a portion of data or it is the tail field. If the number of FLITs is one, the higher part of the current FLIT has the tail payload, where the return link id can be obtained. Otherwise, it will be data coming from a WRITE or ATOMIC request. In this case, the request_decoder module stores all following data chunks in the respective data_request_buffer (each memory controller has it private set of bank operations and data request buffers) until the final FLIT arrives. When this happens, the returning link id can be extracted, and finally, all pieces of information related to the request were obtained. Then, the request command is stored in the bank_request_buffer, and the memory controller can start working.

\footnotetext{
${ }^{3}$ For a more detailed exploration of the available bank level parallelism in HMC devices, we conduct the reader to (ROSENFELD, 2014).
} 


\subsubsection{Memory and TSV Controllers}

Figure 4.9 illustrates the memory controller and the TSV controller modules. The memory controller reads the current request at the top of the bank_request_buffer and generates a sequence of DDR commands that are sent to the memory banks via the TSV bus. The module is implemented as an FSM. When a new request is available, the memory controller reads the target bank address, the operation (READ/WRITE/ATOMIC), the operation size, the memory address, and the memory offset from the buffer into local registers. The memory controller follows the same path as presented in Section 2.1.3. Then, it decodes the bank id and sends the CS command to the destination bank. After that, the memory controller issues a RAS command followed by the row address to bring data from the rows to the row buffer at the selected bank. After the row-to-column delay (tRCD) has passed, the memory controller can start reading/writing data from/to the banks. Then, the memory controller first selects the portion of data within the row buffer that is related to the request by issuing a CAS command, following the column address. At this point, the memory controller can start transferring data to the bank, in the case of a WRITE request (issuing a WE command), or from the bank in the event of a READ request (issuing an OE command). The memory offset value is used to indicate the start position within the row buffer the operation should start being performed. This value is incremented by one until the total request size has been performed. Since the TSV width is limited, potentially several chunks of data need to be transmitted between the memory banks and the memory controller's queues (up to 8 transmissions to move 256 bytes of data). Data from a READ request is stored into the data_response_buffer, while data from a WRITE request is read from the data_request_buffer. The memory controller controls both buffers.

The TSV is a bi-directional vertical bus with a width of 32 bytes that connects the memory banks to the Vault Controller. Since the memory controllers work in parallel and do not share requests' information between them, there must be a module that controls the flow of data and commands through the bus. In consequence, the TSV controller is responsible for monitoring the TSV traffic. The module is connected to all memory controllers, which share information regarding the requested bank address, the size of the request, and the operation being performed. The module implements a round-robin mechanism to select which memory controller has the ownership of the bus. Also, based on the current memory operation being executed, the controller selects the appropriate direction of the bus, allowing data to navigate to the bank or from the bank. 
Figure 4.9: Block diagram of the Memory and TSV controller modules.

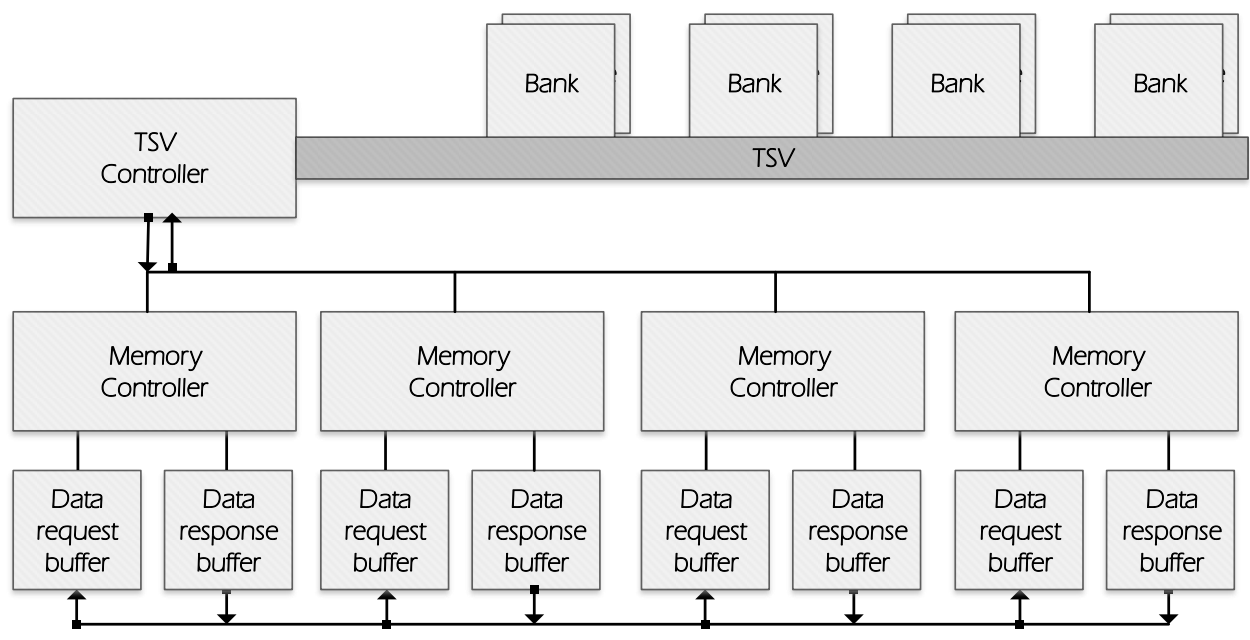

Source: Provided by the author.

\subsubsection{Native PIM}

As previously mentioned, the HMC device is natively capable of executing some arithmetic operations, like addition and bitwise logic operations. All these operations are performed over 8 bytes or 16 bytes of data. Also, they are atomic read-modify-operations, meaning that they operate over a single memory address. To provide these capabilities, we implemented a simple Arithmetic Logic Unit (ALU), which is controlled by one of the memory controllers. Therefore, all native PIM requests are sent to the same memory controller by the request_decoder module.

Figure 4.10: Block diagram of native PIM module.

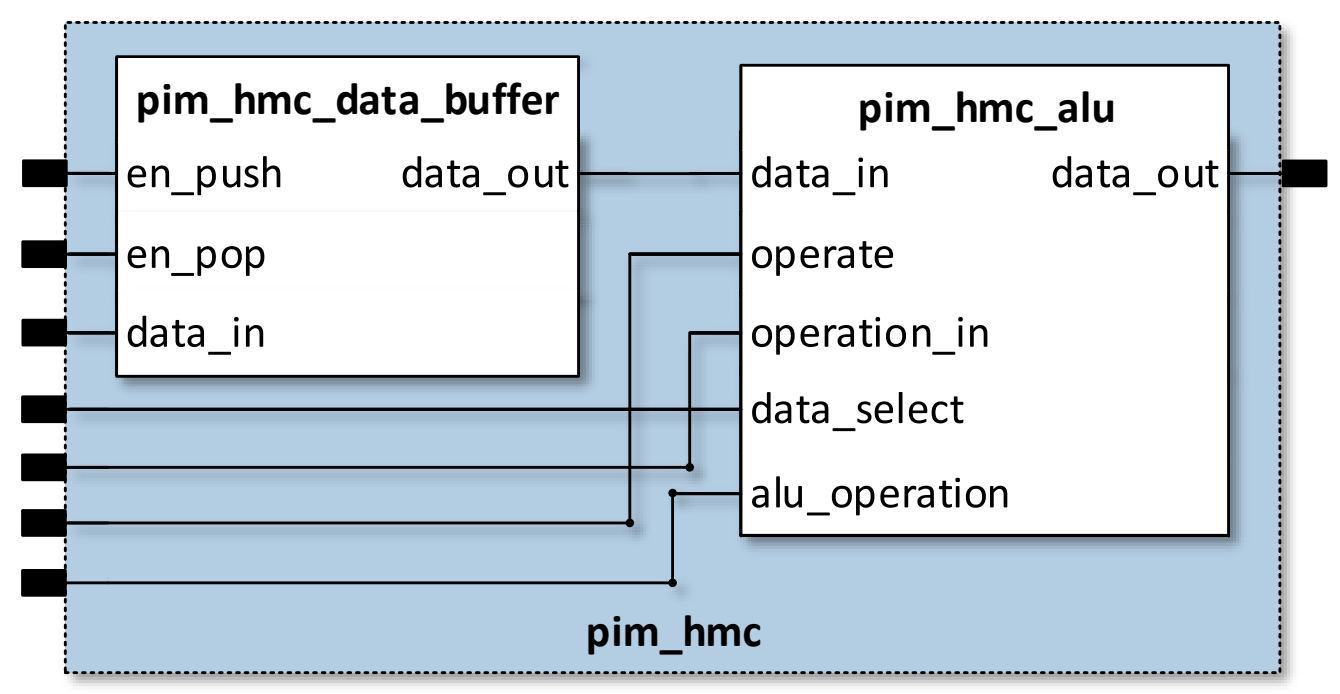

Source: Provided by the author. 
Figure 4.11: Block diagram of the Vault Response module.

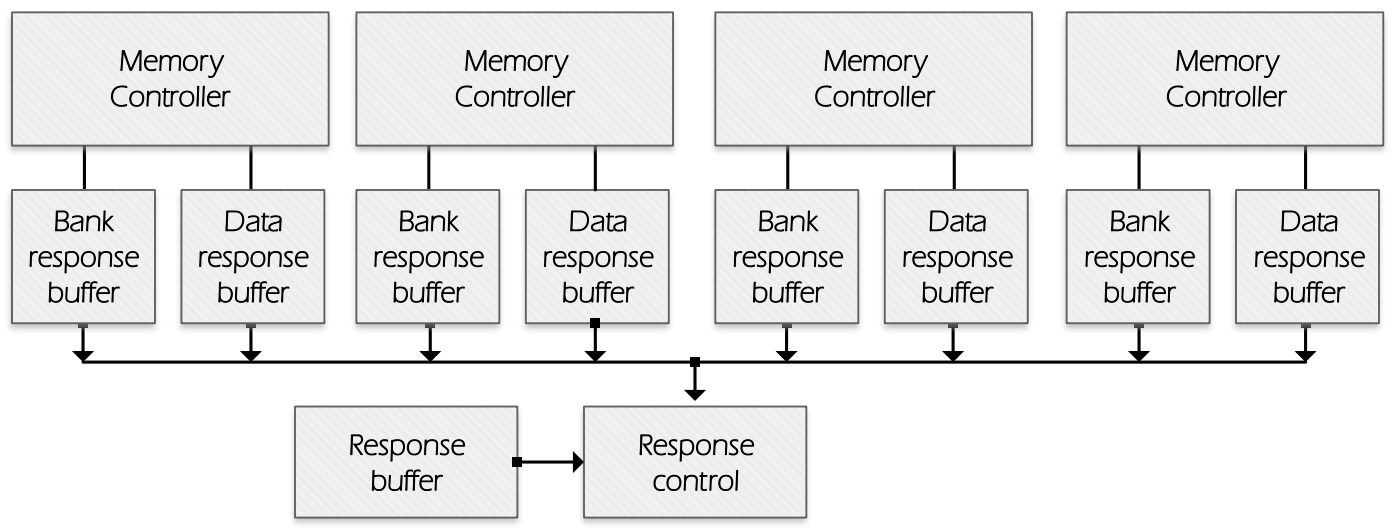

Source: Provided by the author.

Figure 4.10 illustrates the pim_hmc module, which is composed of a local data buffer and a simple ALU. Any native PIM instruction follows three steps during their execution. First, when the instruction arrives at the memory controller, the module issues a READ command to the bank. However, instead of storing the read data into the data_bank_buffer, the data is sent to the pim_hmc_data_buffer. Since the maximum size of a native PIM instruction is 16 bytes, the pim_hmc_data_buffer has this same length. Second, the memory controller issues an execute command to the pim_hmc module by setting the operate signal to high. With this signal, the memory controller also sends the required ALU operation that will be performed, the portion of the data from the data_buffer that should be read (in a case of an eight bytes request), and any immediate value that came with the request package. Finally, after executing the atomic instruction, the memory controller sends a WRITE command bank to the bank, with the result data from the operation. Different from a standard WRITE request, instead of sending data from the bank_request_data_buffer to the bank, the memory controller sends the resulted computation that the pim_hmc module outputs back to the memory bank.

\subsubsection{Response}

Figure 4.11 illustrates the Vault response mechanism and its internal modules. The response_control module is the principal component of the response mechanism, being responsible for scheduling response packets based on the request order, encapsulating the response packet into FLITs, and sending the FLITs back to the Transceiver layer. 
Table 4.2 describes the internal set of input/output ports, methods, and functions present at the response_control module. The module works as follows. First, the prc_response_control() method is called during all positive edges of the clock. This method implements the FSM that controls the scheduling response mechanism. The FSM works as follows. First, at the IDLE state, the method reads the the tag_from_rsp_buffer and the is_pim_instruction fields at the top of the response buffer. The first signal tells the current tag that must be serviced by the response mechanism, and the second indicates if this tag is related to a custom PIM instruction. All this information was stored at the response_buffer by the request_decoder module at the moment the request packet arrived at the Vault Controller. If the current response tag is related to a custom PIM instruction, the FSM can follow to the next state. Otherwise, it needs to check the tag at the top of each bank_response_buffer to identify which memory controller is servicing the request related to the current tag. After that, the FSM needs to wait for this particular memory controller to raise the bank_status signal, indicating that it has finished servicing the request. Then, the FSM reads relevant pieces of information from the specific memory controller bank_response_buffer, as the number of FLITs within the response packet, the link the response must travel, the cub id of the response, and the command that came with the request packet. After that, the FSM can raise the $r s p \_w i l l \_s e n d$ signal indicating to the memory controller that its request was chosen to be serviced by the response module. Finally, the FSM transitions from IDLE to the SENDING state. At this state, the FSM starts encapsulating the response packet. First, it creates the header portion of the response FLITs. If the number of response FLITs is one, it also creates the tail portion of the FLIT and stores the produced FLIT into the local host_response_buffer. Otherwise, it needs to read the response data from the specific data_response_buffer, encapsulate it into the FLIT, and store the produced FLIT into the host_response_buffer. The process of reading data from the data_response_buffer is repeated until the number of response FLITs of the response was obtained. When that happens, the FSM can go to the FINISHED state, when it sends a pop signal to the response buffer, and the next response tag can be serviced. This described process is performed when the response_dst input signal is equal to zero, indicating that the request packet came (and must return) to the host. If this signal is equal to one, the request came (and must return) from the PIM Interface. In this case, the buffer that the FSM stores the produced FLITs would be the pim_response_buffer. At the end of the FINISHED state, the FSM return to the IDLE state.

The prc_response_control_read_host() method runs at all positive edges of the 
Table 4.2: Description of th response_control module, its input/output ports, methods, and functions.

\begin{tabular}{|c|c|c|c|}
\hline Inputs & Outputs & Methods & Functions \\
\hline $\begin{array}{c}\text { bank_status } \\
\text { en_pop } \\
\text { en_pop_from_pim } \\
\text { is_pim_instruction } \\
\text { tag_from_bank_rsp_buffer } \\
\text { tag_from_rsp_buffer } \\
\text { data } \\
\text { link_from_pim_rqst } \\
\text { response_dst } \\
\text { link } \\
\text { cub } \\
\text { response_lng } \\
\text { cmd }\end{array}$ & $\begin{array}{c}\text { packet_out_host } \\
\text { packet_out_pim } \\
\text { packet_available_host } \\
\text { packet_available_pim } \\
\text { en_pop_bank_response_buffer } \\
\text { rsp_will_send } \\
\text { en_pop_data_buffer } \\
\text { en_pop_response_buffer }\end{array}$ & $\begin{array}{l}\text { prc_response_control } \\
\text { prc_response_control_read_host } \\
\text { prc_response_control_read_pim }\end{array}$ & $\begin{array}{c}\text { creat_header } \\
\text { creat_tail } \\
\text { creat_data } \\
\text { creat_response_packet }\end{array}$ \\
\hline
\end{tabular}

Source: Provided by the author.

Transceiver clock. This method is responsible for reading the FLIT at the top of the host_response_buffer, and writing its value to the packet_out_host output signal. It also raises the packet_available_host signal, indicating that there is a new response FLIT available to the Transceiver Layer. On the other hand, the prc_response_control_read_pim() method runs at all positive edges of the PIM Interface clock. It has the same functionality of the prc_response_control_read_host() module, but it reads the FLITs from the pim_response_buffer, and sets the packet_out_pim and packet_available_pim output ports.

\subsubsection{PIM Interface}

The primary goal of this work is to provide a simple yet concise infrastructure that allows new PIM architecture exploration. To do so, we have implemented the HMC design as previously described, and studied a way to insert a custom processing module into HMC without having to worry about HMC organization. We came to a simple PIM Interface, placed into the Vault Controller, that receives custom PIM instructions, and also provides a mechanism to perform read/write operations.

To implement the PIM Interface, we decided not to modify dramatically the HMC data-path and its communication protocol. However, we still had to perform four minor modifications to the HMC design. First, we have included a new opcode command to the set of HMC command operations. The opcode included was the PIM_INSTRUCTION. 
Figure 4.12: Block diagram of the proposed PIM Interface.

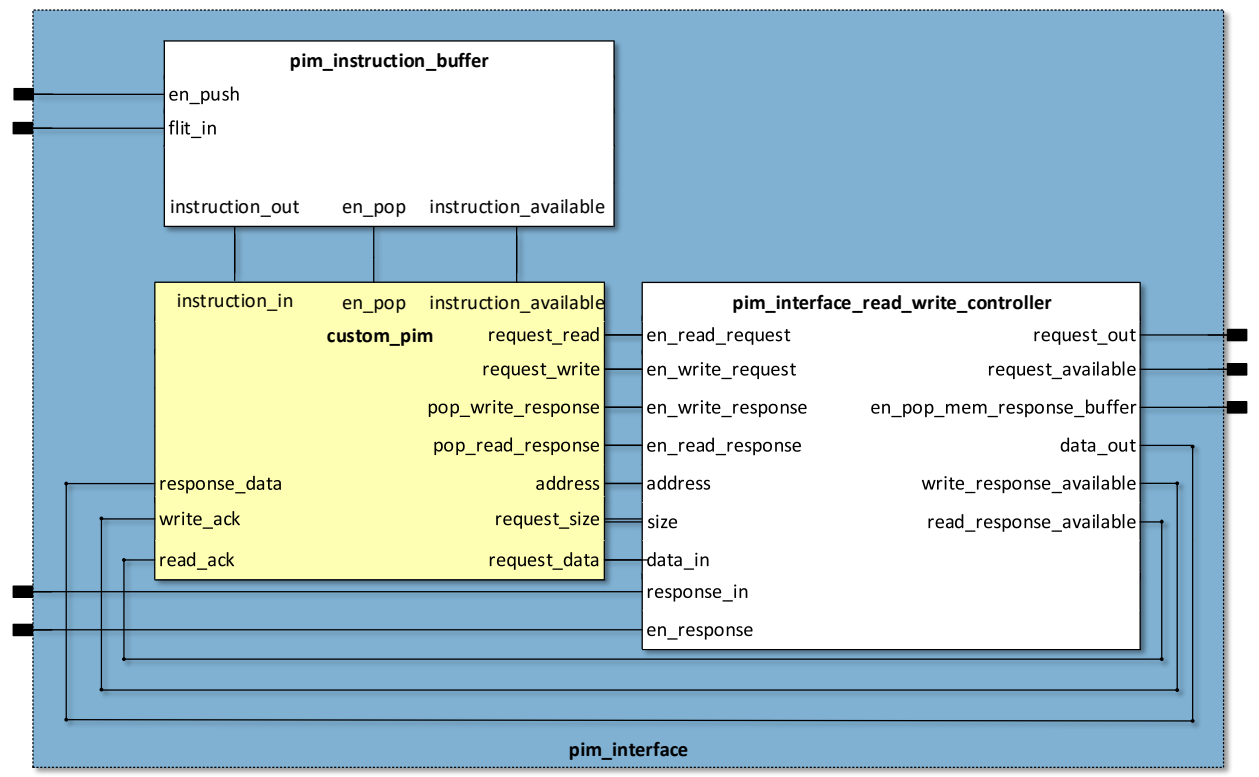

Source: Provided by the author.

All custom PIM instructions will make use of this same opcode. A single PIM request is seen by our simulator similarly to a 16 bytes write request. All information related to the custom PIM instruction will be placed in the data sections of the request packet. Therefore, the user does not need to worry about violating already reserved opcodes or being limited by the number of free available HMC opcodes when designing his or her instruction set. Second, we have included the already mentioned traffic_request_controller module. This module is required to allow a request flow to come from both the host and from the PIM Interface. Third, we have made use of a reserved bit from the request header field (bit 23), to indicate to the request_controller whether the current READ/WRITE request should return to the host or the PIM Interface. Finally, we have included a path between the request decoder and the PIM Interface, therefore FLITs related to a PIM request can be sent to the PIM Interface.

Figure 4.12 depicts the proposed PIM user interface. The module is composed of the pim_instruction_buffer and the pim_interface_read_write_controller. The process of performing custom PIM operations works as follows. First, when issuing a new request to the Transceiver module, the user must specify the PIM_INSTRUCTION opcode, the address of the request, and then a 16 bytes of data that represents their custom instruction. The packet_generator will encapsulate this request the same way it makes a write request. After that, the produced FLITs will travel through the same path as a standard 
request until arriving at the request_decoder. At this point, when the request_decoder de-codifies the command portion of the request, it will not recognize the opcode, and then will send the upcoming FLITs to the pim_instruction_buffer at the PIM Interface. The pim_instruction_buffer will then remove additional payloads of the FLITs (as header and tail fields), and will raise the instruction_available output signal to notify the custom_pim module that there is a new instruction currently available to it. The custom_pim does not need to have any information about how the packet was encapsulated since the upcoming PIM instructions have only information related to its own instruction set.

When the custom_pim instruction decides to perform a READ or WRITE operation to the HMC module, it sets the request_read or request_write signal, informing to the pim_interface_read_write_controller that a new HMC request must be generated. The custom_pim also provides the address it wants to access, the size of the request, and data values in the case of a WRITE operation. The controller will then encapsulate the PIM request into FLITs, creating a header and tail field in accord with the HMC protocol, and then transmitting the generated request back to the Vault Controller. Also, it will set bit 23 of the header field to one, indicating that the response package must return to the PIM Interface. The request will be sent to the traffic_request_monitor, and when appropriated, it will be sent to the request_decoder. Once at the Vault Controller, the request will follow the same path as any standard host request. However, once the request goes through the response_control, the module will check that the rsp_destination signal is set to one (recall this signal stores the value of bit 23 of the header), and then all response FLITs will be sent back to the PIM Interface. Finally, when the PIM Interface receives the response packets from the Vault Controller, it will notify the custom_pim module that its READ/WRITE request is completed by raising the write_ack or read_ack signals. In the case of a READ request, the PIM Interface removes the header and tail payloads of the response package, and send the requested data to the custom_pim.

\subsubsection{Bank}

The Bank module replicates the behavior of a DRAM bank, as described in Section 2.1. The module receives the CS, RAS, CAS, OE, WE signal from the memory controller. The memory controller also sends the request address and the row buffer offset to the bank. Finally, data can traffic through the Vault Controller and the banks, and vice-versa. 
Figure 4.13: Block diagram of the Traffic Monitor class.

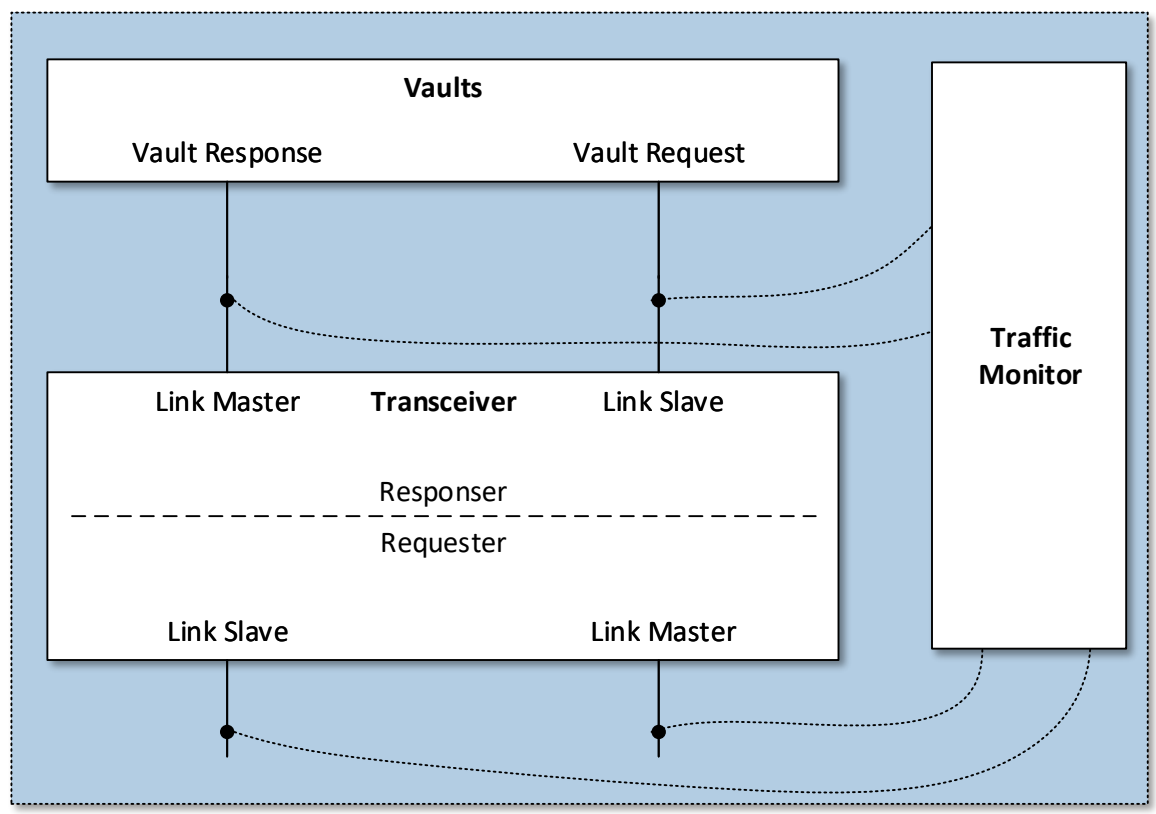

Source: Provided by the author.

Each HMC bank stores 16 Mbytes of data (there are 16 banks per Vaults, and 32 Vaults, summing up 8 Gbytes of memory). To reduce the amount of data that the simulator has to allocate to each bank, we have implemented the 2D grid of DRAM cells using the $\mathrm{C}++$ standard hash data structure. In this way, we could reduce the amount of memory that our simulator will allocate during simulation. In other words, our simulator will only allocate all 8 Gbytes of memory when all bank addresses, from all banks, in all Vaults are currently being accessed. To perform a READ/WRITE operation, the bank module goes through the following states. First, when a CS signal arrives, the bank will decode this signal to check if the upcoming request targets itself. If it does, the bank will go to the activated state. Second, when the RAS signal is received, the bank loads the data stored in the hash in the request address into the row buffer. Third, when the CAS signal arrives, the bank will select the section of the row buffer related to the received column address. Fourth, when receiving a WE signal, the bank reads data from the TSV bus and writes it back to the row buffer. If it receives an OE signal, it writes the row buffer value to the TSV bus. Finally, at the arrival of a DONE signal, the bank writes back the row buffer value at the hash. 


\subsection{Traffic Monitor}

The Traffic Monitor class is responsible for tracking the request and response flux of packets in both Transceiver and Vault modules. Figure 4.13 illustrates how the traffic monitor is connected to the system. The class has internal variable counters that store the number of packets transmitted, the total number of bytes written/read by the host, the total number of READ/WRITE instructions, and the total time spent during run-time. ${ }^{4}$ To connect the traffic monitor to the rest of the HMC design, a pointer to the class is stored in some key $h m c \_d e v i c e$ modules. These modules are the link_master and link_slave modules of both Requester and Responder Layers. Also, we connected each Vault's request and response modules to the traffic monitor class. In this way, we can check the external Link bandwidth and internal Vault bandwidth.

The monitoring process works as follows. When creating a new request packet, the Requester Layer informs the Traffic Monitor the size of the request in bytes and its type (READ/WRITE). If this is the first package being created, the Traffic Monitor sets its timing counter. Then, every time the request passes through one of the modules that have a Traffic Monitor pointer, it writes the current timing stamp back to the Traffic Monitor. The timing stamp depends on the clock of the module. Also, it can depend on specific timing parameters (as the DDR timings). To end the simulation, the Traffic Monitor also checks if and request_global_tag buffer at the Request Layer is empty, what indicates that all request packets have already been returned to the host. Also, it checks if the custom_pim is not executing any instruction. If both conditions are true, the Traffic Monitor can end the simulation and finish to count the execution run-time.

\footnotetext{
${ }^{4}$ It is important to differentiate the total simulation time and the final run-time. The former one tells the time the simulator spent running on the user machine. The latter informs the time the proposed HMC model took to service all host requests.
} 


\section{SIMULATION MECHANISM}

In this section, we explain the steps to produce a simulation using the proposed framework. There are eight important files related to the simulation process. First, since our simulator is trace-based, we provided some scripts that can produce valid trace files to feed the simulator. There are four distinct trace scripts available. The trace-generatorseq.py script generates READ/WRITE requests that accesses memory sequentially. The trace-generator-pim-seq.py script also generates a trace file that access memory sequentially, but creates native PIM HMC requests. On the other hand, both trace-generatorrand.py and trace-generator-pim-rand.py scripts generate READ/WRITE and PIM HMC requests, respectively, that access memory randomly. The trace file generated has the memory address, HMC command, cub_id, and in the case of a WRITE request, the input data. Besides that, the defines configuration file specifies some parameterizable HMC values. The user can modify the number of Vaults, the number of links, the row buffer size, the TSV width, the number of memory controllers, the DRAM's timing parameters, and the Vault and Transceiver clock rate in the defines file. Meanwhile, the hmc-testbench file drives and monitors the hmc-device module. As previously mentioned, the trafficmonitor gathers run-time statistics. The hmc-device is the implementation of the HMC architecture. Finally, the simulator outputs the simulation results and simulation statistics files.

Figure 5.1 illustrates the simulating steps that CLAPPS goes through during exe-

cution. In 1 , the user generates the trace file that will be used during simulation. The trace file can be produced using any of the available trace generator scripts. However, if the user decides to evaluate the memory execution of a real benchmark, he or she needs to convert the benchmark's memory access pattern to the simulator's trace format. Each line of the trace file has the HMC opcode, the memory address, the number of data FLITs in the request, and the list of data itself. For example, in the following line of a trace file,

\section{9}

the first seven bits (0001001) indicate the operation is a WRITE request of 32 bytes, the following 34 bits indicate the target memory address, then the third portion indicates that the list of input data has two elements, and then 7 and 9 are the input data elements themselves.

In 2 the user can modify the standard HMC structure by changing the define file. Then, he or she needs to compile the simulator. Since SystemC is based on $\mathrm{C}++$, the 
Figure 5.1: Simulation steps our mechanism travels through execution.

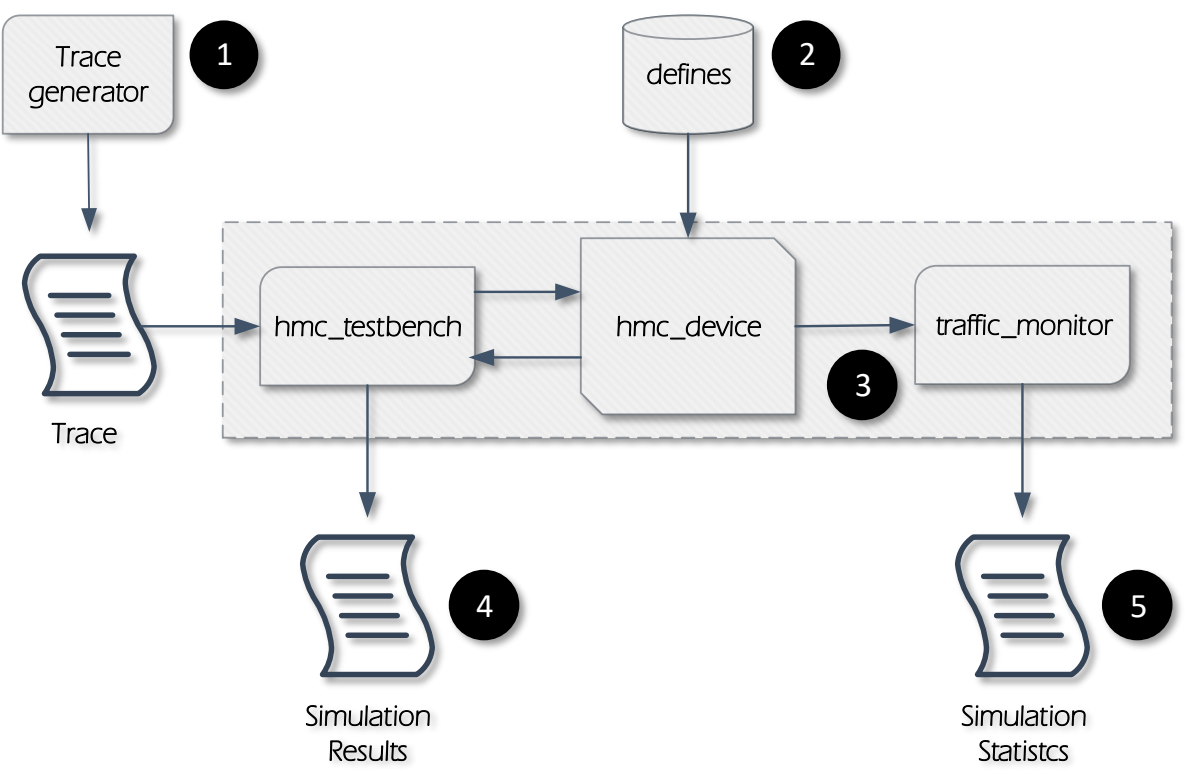

Source: Provided by the author.

only library the user needs to provide and link during compilation is the SystemC 2.3.1. one. When the compiling and linking process has finished, the user can run the simulator. 3 describes the run-time process. The hmc-testbench will read the trace file, driving the $h m c$-device with all requests in the trace file. The requests are sent to the hmc-device one per clock. Besides that, the hmc-testbench will monitor the output port of the hmc-device, writing all response packets generated by the latter into the simulation results file (4) To check the correctness of the simulation, the user can compare the simulation result file with the gold file produced by each one of the four trace generator scripts. Finally, at the end of the simulation, the traffic-monitor writes the produced statistics to the simulation statistics file 5. This file is divided into per Vault and per Link statistics. In both cases, it is shown the total number of bytes and written, the total time (in ns) the hmc-device took to perform all requests, the obtained individual and global bandwidth (in GB/s), and the number of packets produced. 


\section{EXPERIMENTAL SETUP AND RESULTS}

In this section, we will show the simulation potential provided by our mechanism. All our experiments target the entire vault and link bandwidth that we were able to extract during simulation. To simplify the analysis, we have divided our results in three categories: HMC Memory Validation, HMC Atomic Requests, and PIM Implementation. Table 6.1 describes all configured parameters used in all presented results. All these parameters can be modified as needed.

\subsection{Memory Validation}

Our first concern when building the simulator was to create an architectural HMC design that would also work as a simple memory simulator. However, since there is no public information about the HMC internals or even about the DDRx memories that composes the design, we have used as an implementation guide published related work, in special the work presented by (ROSENFELD, 2014).

First, we were interested in investigating if there was a significant performance different when comparing read, write, and read+write requests, as cited in (ROSENFELD, 2014). Therefore, we have run $8 \mathrm{~K}$ read and write requests for various row buffer sizes. All the requests match the row buffer size. Therefore we could extract maximum performance from the current simulation. We have used a $50 \%$ read/write ratio in our testbench.

Table 6.1: HMC configuration.

\begin{tabular}{ll}
\hline Number of Vaults & 32 \\
Number of Links & 4 \\
Banks/Vault & 16 \\
Lane Bandwidth & $30 \mathrm{Gbs}$ \\
Memory Size & $8 \mathrm{~GB}$ \\
Burst Width & $8 \mathrm{~B}$ \\
Number of DRAM Dies & 8 \\
RCD Latency & $10.4 \mathrm{~ns}$ \\
DRAM Frequency & $166 \mathrm{MHz}$ \\
Row Buffer & $32,64,128,256 \mathrm{~B}$ \\
Row Policy & Close-row \\
\hline
\end{tabular}

Source: Provided by the author. 
Figure 6.1: Link and Vault total bandwidth for sequential read and write requests.

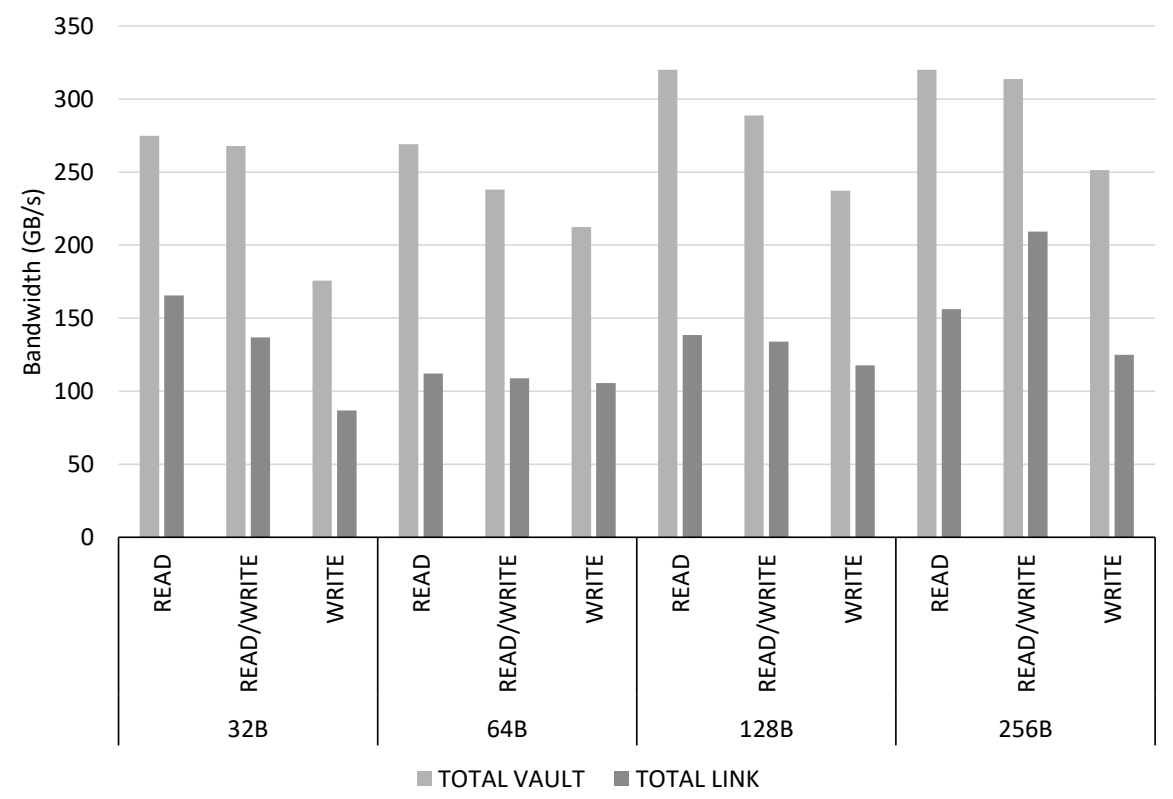

Source: Provided by the author.

Besides that, the request addresses were generated aiming to evict link/vault/bank conflicts. Figure 6.1 shows the results of the simulation. One can notice that read requests are faster than write requests when considering the total vault bandwidth. That happens because, from the DDR point of view, a read request loads data from the memory cells, stores the data into the row buffer, to then be read out by the memory controller. On the other hand, write requests will generate the same process, and also it will need to write user data to the row buffer, to then, be stored back into the memory cells. To summarize, a

Figure 6.2: Vault total bandwidth for sequential read requests.

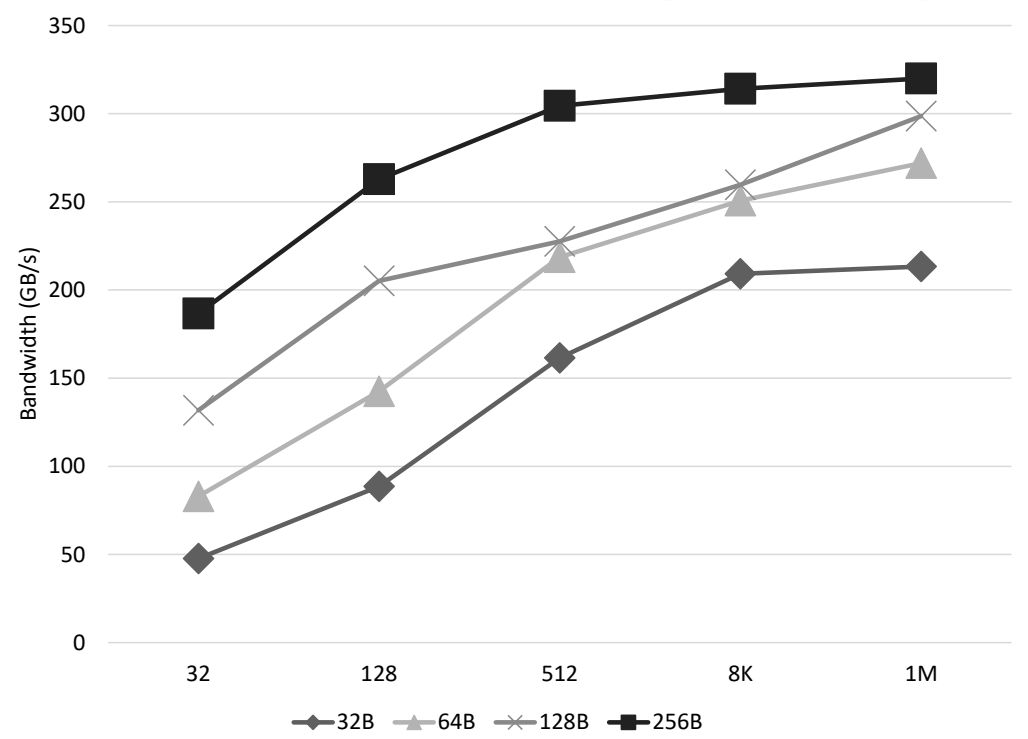

Source: Provided by the author. 
Figure 6.3: Link total bandwidth for sequential read requests.

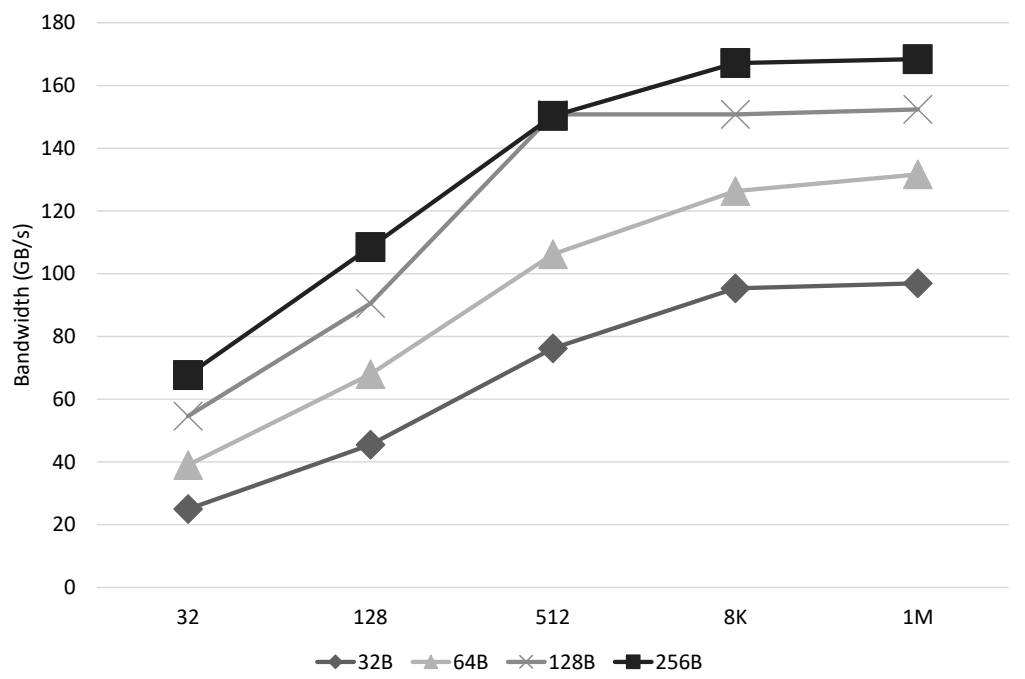

Source: Provided by the author.

write request is slower than a read request because a write command generates a sequence of reading and writing requests. However, this observation is not valid for the links. In general, the maximum link bandwidth will be achieved when a mix up of reading and writing request occurs. That behavior is caused by the fact that the overhead to send the read request to the memory or to send the acknowledge packet back to the host, in the case of a write request. This observation agrees with the one presented in (ROSENFELD, 2014).

Next, we have focused on obtaining the maximum vault bandwidth that our simulator can provide. To do so, we have simulated five different scenarios with 32 to $1 \mathrm{M}$ read instructions while varying the row buffer size. This number of requests was chosen because with 32 requests one could measure the bandwidth in the case of only one request per vault; with 512 requests one can understand the available vault parallelism when all banks of all vaults receive a single request; with $1 \mathrm{M}$ requests, one could saturate the memory performance. 128 and $8 \mathrm{~K}$ requests were used as intermediate points. Figure 6.2 and Figure 6.3 show the vault and link performance results respectively. It is evident that the biggest the row buffer size, the better the achieved bandwidth. This result is explained because the vault controller works in a pipeline fashion. Once a bank row buffer has been opened, the only performance limitation would be receiving or sending data from/to the bank unit through the TSV. However, since DDRx memories reply to requests in bursts of data, this extra latency to access the TSV is reduced. The maximum bandwidth CLAPPS could achieve $312 \mathrm{~GB} / \mathrm{s}$, with row buffer size of $256 \mathrm{~B}$. 
Figure 6.4: Vault total bandwidth for sequential and random atomic requests.

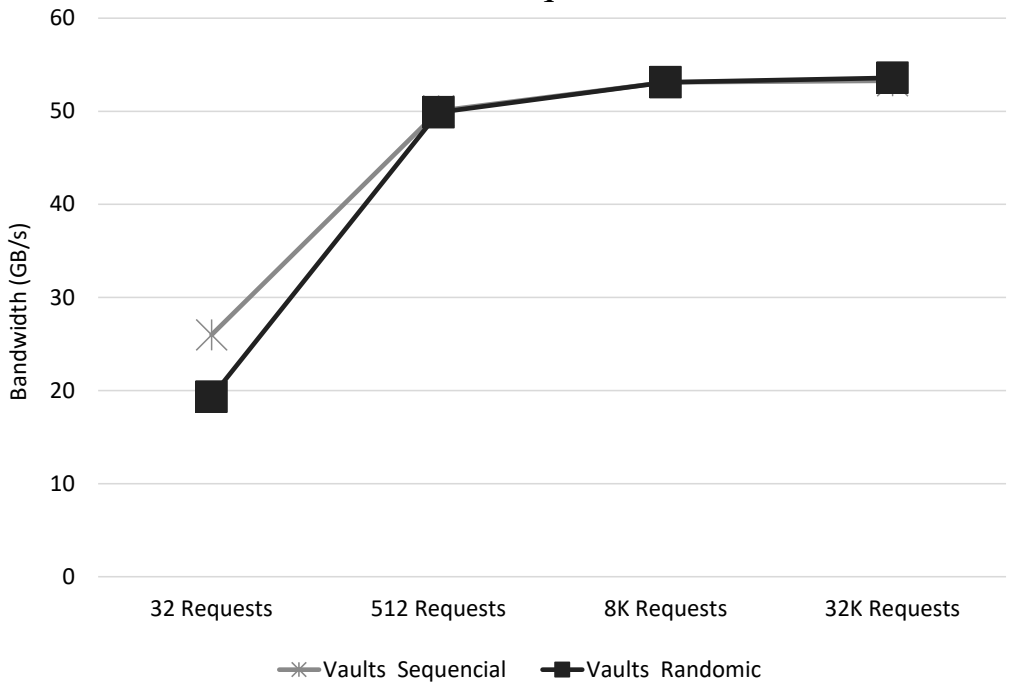

Source: Provided by the author.

\subsection{Atomic Requests}

With our memory architecture validated, we needed to experiment with the HMC atomic instructions. Since all atomic instructions follow the same data path, they all have the same execution latency. Therefore, all our simulation were based on the add dual 8B instruction. Also, we have generated testbenches with random request addresses. Experimenting with a random set of requests is important because HMC devices were first designed to target applications with sparse data accesses. Figure 6.4 and Figure 6.5 show our simulation results. The results for the random-based requests were obtained

Figure 6.5: Link total bandwidth for sequential and random atomic requests.

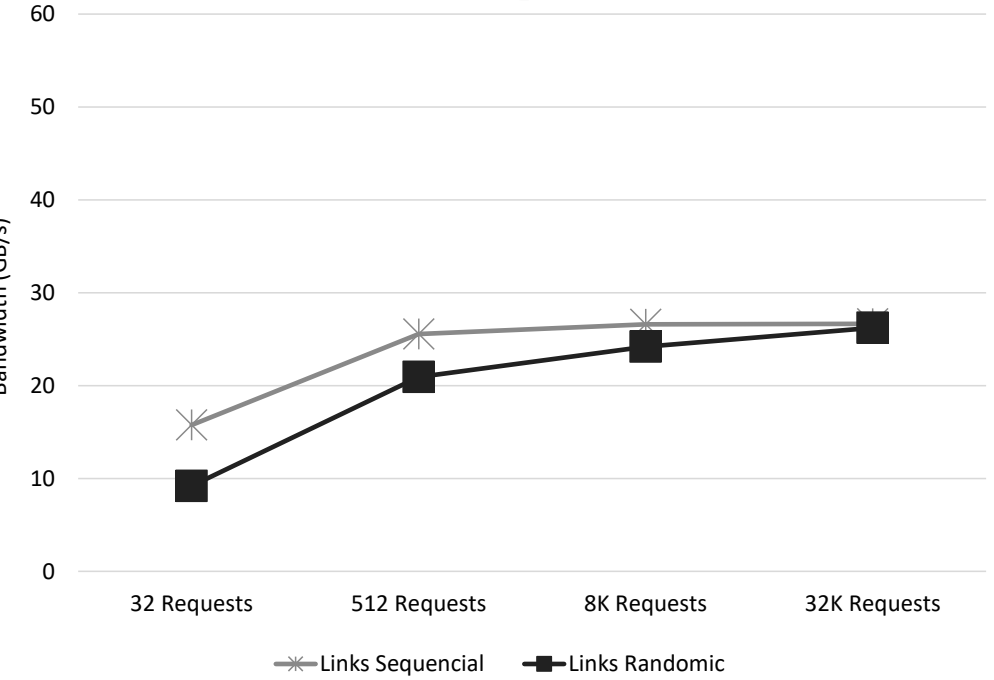

Source: Provided by the author. 
with three different set of inputs. Some observations can be pointed out by these results. The first observation one could make is that atomic operations provide significative lower bandwidth than reading or writing requests. The bandwidth reduction happens because a single atomic request will generate a sequence of reading and write requests. Besides that, only one ALU is available to execute the instruction, therefore limiting the vault parallelism. Secondly, it is possible to notice that unpredicted access patterns do not severely prejudice the bandwidth for random requests.

\subsection{Case of Study: PIM Interface}

To test the effectiveness and usability of our PIM Interface, we included into CLAPPS, using the provided set of resources, the PIM architecture developed by (SANTOS et al., 2017).

RVU is a reconfigurable accelerator that targets vector operations with varied operand sizes. Figure 6.6 illustrates the internal organization of (SANTOS et al., 2017). It is an improvement over the work proposed by (ALVES et al., 2016) that aims to reduce unnecessary data movement from memory to the accelerator device. One RVU device was inferred inside each vault module. A single accelerator has a set of 8 registers with up to 256B each, 32x64 integer, and floating-points Functional Units (FUs), and operates

Figure 6.6: Overview of the Reconfigurable Vector Unit (RVU) organization.

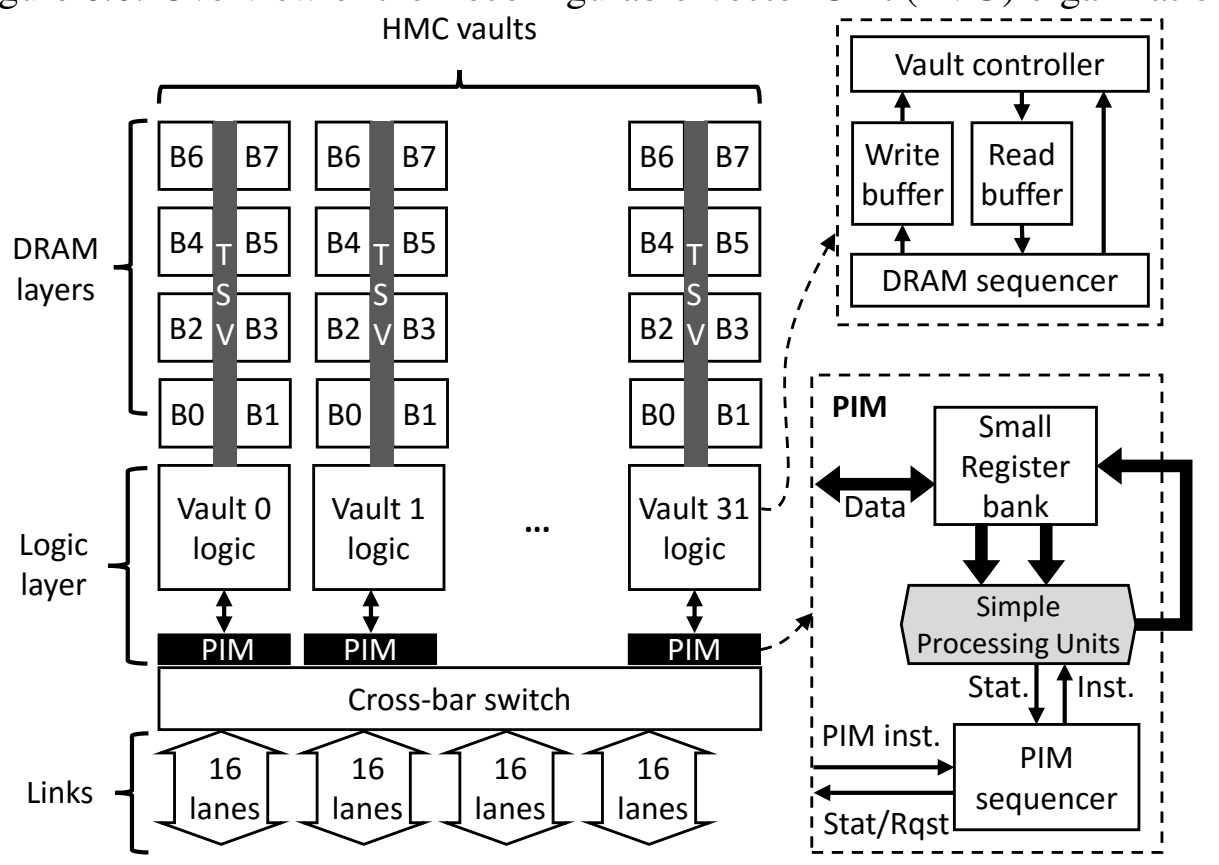

Source: (SANTOS et al., 2017). 
Figure 6.7: RVU's FSM

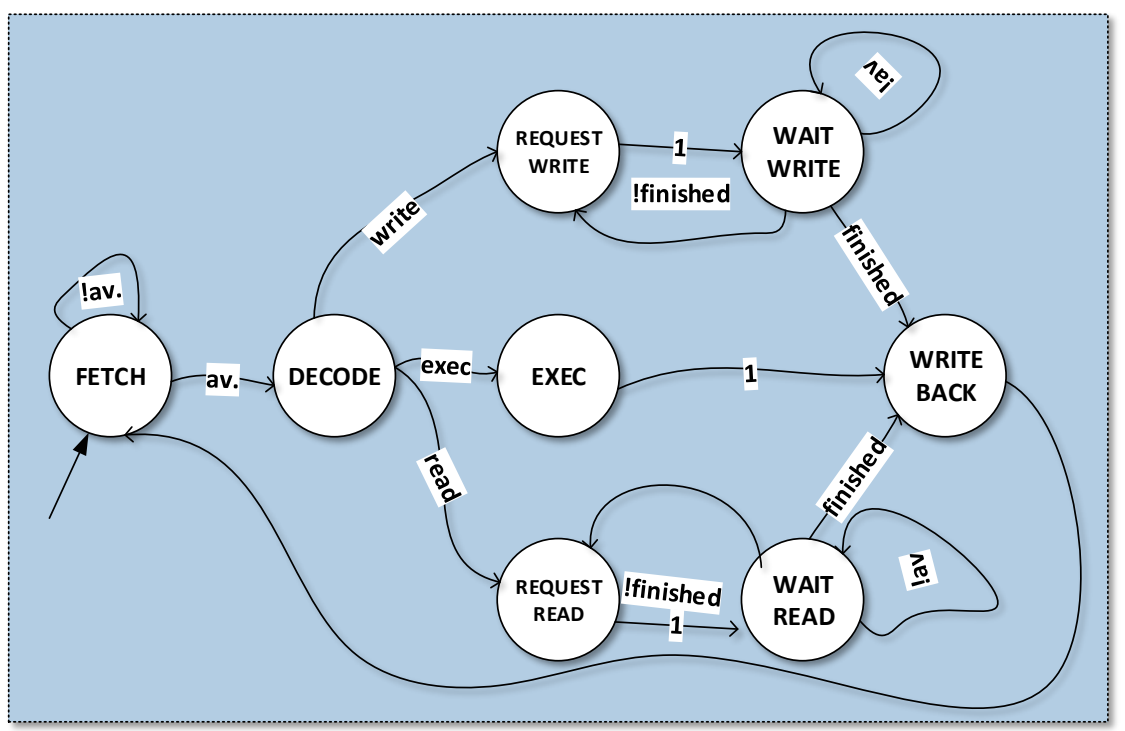

Source: Provided by the author.

at the same frequency as the vault controller. Besides that, each device can operate over a maximum of $256 \mathrm{~B}$ at the time; therefore all accelerators together being able to execute an $8 \mathrm{~KB}$ vector operation at a given time.

To implement the (SANTOS et al., 2017) architecture into our simulator, we included two new files: the $r v u \_d e f i n e s$ and the $r v u \_i m p l e m e n t a t i o n$. The first one defines the accelerator's Instruction Set Architecture (ISA), the number of FUs, and the number and width of the register file. The second one is the actual implementation of the proposed RVU architecture. We have developed RVU as a simple FSM, which is illustrated in Figure 6.7. While in the initial state, FETCH, RVU waits until there is a new instruction available. When this happens, RVU reads and stores the instruction into the instruction register, then sending a pop signal to the pim_interface to pop the instruction out the instruction_queue. Then the FSM goes to the DECODE state, where it decodes the instruction in accord to its ISA defined at the $r v u \_d e f i n e s$ file. Once the instruction has been decoded, the FSM can go to either the REQUEST_READ, REQUEST_WRITE, or EXEC states, depending on the instruction type. If it goes to the REQUEST_READ, it means data needs to be loaded from memory. To do so, it writes the required memory address, the size of the request, and rises the en_read_request signal, indicating to the pim_interface that a new request needs to be serviced. Then the FSM goes to the WAIT_READ state, where it waits until the pim_interface raises the read_response_available signal, indicating that the data from memory is available. When this happens, the machine goes to the 
WRITE_BACK state, where it stores the returned data into the register indicated by the instruction, and sends an acknowledge signal back to the pim_instruction, indicating it can pop the data out its data queue. This process is repeated until all requested data has been returned from memory and stored in the vectorial register. Then, the FSM goes back to the FETCH state to wait for new instructions. In the case of a WRITE instruction, the FSM passes through the same states. However, it provides the data from a register to be written back to memory. In the EXEC state, RVU performs actual computational, in accord to the instruction received.

According to (SANTOS et al., 2017), when the user configures their mechanism to run over its maximum operation size, it will achieve similar results to the work of (ALVES et al., 2016). Besides that, from all benchmarks executed by (ALVES et al., 2016), the vec-sum algorithm provided a maximum bandwidth exploration from memory. Thereby, since our evaluation metric is vaults and links total bandwidth, we have decided to perform this same benchmark in our experiment.

In the results provided by (ALVES et al., 2016), the total vault bandwidth obtained for a vec-sum operation was $315.9 \mathrm{~GB} / \mathrm{s}$. There is no information in (ALVES et al., 2016) regarding of the total link bandwidth. In our simulation, we have obtained similar results. In total, the vault performance was $317.8 \mathrm{~GB} / \mathrm{s}$, and the link performance was 213.36 $\mathrm{GB} / \mathrm{s}$. 


\section{CONCLUSIONS AND FUTURE WORK}

In this work, we presented CLAPPS, a generic Cycle Accurate Parallel Processing In Memory Simulator. Our simulator provides an interface to implement PIM architectures targeting new 3D-stacked memories devices, in particular, the HMC architecture. CLAPPS has been built using the SystemC programming language since it can provide the flexibility of a high-level programming language while generating a final design description similar to the one an HDL language would produce. We have demonstrated that our memory model can achieve closer to the total amount of bandwidth cited by the HMC consortium. Moreover, we have shown with a case of study, how our PIM interface can be useful to the final user.

Table 7.1 compares the simulators discussed in Section 3 with the proposed simulator. First of all, it is important to point out that, similar to the other simulators, CLAPPS is also cycle-accurate and implements the native HMC atomic instructions. It provides a simple mechanism to include novel PIM architectures into the simulator while achieving the theoretical HMC peak bandwidth of $320 \mathrm{~GB} / \mathrm{s}$. Besides that, CLAPPS deals with parallelism natively by making use of SystemC's concurrent execution model. Finally, our simulator is fully parameterizable.

There are some known limitations of our simulator. First, it is the fact that our simulator is trace-based. It means that the memory footprint generated by an application needs to be recorded during run-time and then read back into our simulator off-line. Even though this is a common practice of memory simulators, it might include some error to the simulation results, since online behaviors, as changes in context or interrupts, are

Table 7.1: Comparison between available PIM simulators and CLAPPS. "?" indicates the information could not be obtained.

\begin{tabular}{|c|c|c|c|c|c|c|c|c|}
\hline Simulator & $\begin{array}{l}\text { Simulation } \\
\text { Accuracy }\end{array}$ & $\begin{array}{l}\text { Native PIM } \\
\text { Capabilities }\end{array}$ & $\begin{array}{l}\text { Novel PIM } \\
\text { Capabilities }\end{array}$ & $\begin{array}{l}\text { Peak } \\
\text { Bandwidth }\end{array}$ & $\begin{array}{l}\text { Deal with } \\
\text { Parallelism }\end{array}$ & $\begin{array}{l}\text { Input } \\
\text { Type }\end{array}$ & $\begin{array}{l}\text { Parame- } \\
\text { trizable }\end{array}$ & $\begin{array}{l}\text { Simulation } \\
\text { Time }\end{array}$ \\
\hline HMC-SIM & $\begin{array}{l}\text { Cycle- } \\
\text { Accurate }\end{array}$ & Yes & $\begin{array}{l}\text { Yes - } \\
\text { Limited }\end{array}$ & $320 \mathrm{~GB} / \mathrm{s}$ & $\begin{array}{l}\text { Event } \\
\text { queues }\end{array}$ & $\begin{array}{l}\text { Offline } \\
\text { Trace }\end{array}$ & No & $?$ \\
\hline Cas-HMC & $\begin{array}{l}\text { Cycle- } \\
\text { Accurate }\end{array}$ & Yes & No & $?$ & $\begin{array}{l}\text { Event } \\
\text { queues }\end{array}$ & $\begin{array}{l}\text { Offline } \\
\text { Trace }\end{array}$ & No & $28.7 \mathrm{~s}$ \\
\hline SMC & $\begin{array}{l}\text { Cycle- } \\
\text { Accurate }\end{array}$ & Yes & Yes & $205 \mathrm{~GB} / \mathrm{s}$ & $\begin{array}{l}\text { Event } \\
\text { queues }\end{array}$ & $\begin{array}{l}\text { Online } \\
\text { Trace }\end{array}$ & Yes & $4.5 \mathrm{~s}$ \\
\hline CLAPPS & $\begin{array}{l}\text { Cycle- } \\
\text { Accurate }\end{array}$ & Yes & Yes & $320 \mathrm{~GB} / \mathrm{s}$ & Natively & $\begin{array}{l}\text { Offline } \\
\text { Trace }\end{array}$ & Yes & $20 \mathrm{~m}$ \\
\hline
\end{tabular}

Source: Provided by the author. 
not replicated during the off-line simulation. To cope with this problem, we expect to integrate our simulator into the gem5 (BINKERT et al., 2011) infrastructure, since it supports SystemC-based designs. Second, the custom PIM programmability can impose a challenge for novel architectures. Despite the fact that this is not a problem intrinsic of the presented simulator but indeed an issue related to state-of-the-art PIM architectures, this can be a problem when implementing a cycle-accurate detailed model of the proposed PIM design, since probably there will be no compiler to generate code for the design. Some previous works, as (AHN et al., 2015b), have offered to solve this problem by developing PIM architectures with the same ISA as the host device. However, there is a trade-off between flexibility and programmability in this case. Third, data coherence is not guaranteed by our simulator. This problem occurs when both host and PIM are working in parallel, and the PIM generates a READ request, and in the sequence, the host sends a WRITE request to the same memory address. It might happen in this case that the WRITE request to be serviced before the READ request. Then the data read by the PIM will be the newest one, instead of the that was stored in memory at the time the PIM received the READ request. Fourth, CLAPPS's simulation time is orders of magnitude larger than the other simulators. This happens for two reasons. The first one is because the HMC device is highly parallel in its organization. For example, during simulation, only considering the HMC Vaults, 32 distinct modules need to be launched and executed concurrently on a single CPU core. This is similar to simulate 32 CPU cores, which is even a challenge for the most popular architectural simulator, gem5. The second one, and most important, is the fact our simulator implements HMC in a granularity of bits, which is different from the other simulators that use a behavior simulation. With this, our design can be easily converted to an RTL description, allowing to perform logic synthesis directly from our code. Finally, our simulator does not implement the HMC engine that deals with errors correction. We have chosen not to design this part of the $\mathrm{HMC}$ architecture because the primary source of errors in a real HMC device is the one generated by the high-speed serial links. Since we do not run the link lanes at the same high frequency as the actual hardware, an error coming from the links would not happen. Therefore, the error correction mechanism would only slow down the simulation process. In future works, we aim to include statistics about power and energy consumption into our design. 


\section{REFERENCES}

AHN, J. et al. A scalable processing-in-memory accelerator for parallel graph processing. In: 42ND ANNUAL INTERNATIONAL SYMPOSIUM ON COMPUTER ARCHITECTURE (ISCA), 2015, Portland, USA. Proceedings... [S.1.]: IEEE, 2015. p. 105-117.

AHN, J. et al. Pim-enabled instructions: A low-overhead, locality-aware processingin-memory architecture. In: 42ND ANNUAL INTERNATIONAL SYMPOSIUM ON COMPUTER ARCHITECTURE (ISCA), 2015, Portland, USA. Proceedings... [S.1.]: IEEE, 2015. p. 336-348.

ALVES, M. A. Z. et al. Large vector extensions inside the hmc. In: DESIGN, AUTOMATION AND TEST IN EUROPE CONFERENCE (DATE), 2016. Proceedings... [S.1.]: IEEE, 2016. p. 1249-1254.

AMBA, A. Protocol specification v2. 0. ARM Holdings plc Std, 2003.

AZARKHISH, E. Memory Hierarchy Design for Next Generation Scalable Many-core Platforms. Thesis (PhD) - alma, 2016.

AZARKHISH, E. et al. A case for near memory computation inside the smart memory cube. In: INTERNATIONAL WORKSHOP ON EMERGING MEMORY SOLUTIONS, 2016. Proceedings... [S.1.:s.n.], 2016.

AZARKHISH, E. et al. Design and evaluation of a processing-in-memory architecture for the smart memory cube. In: INTERNATIONAL CONFERENCE ON ARCHITECTURE OF COMPUTING SYSTEMS, 2016. Proceedings... [S.1.]: Springer International Publishing, 2016. p. 19-31.

AZARKHISH, E. et al. Neurostream: Scalable and energy efficient deep learning with smart memory cubes. In: ARXIV PREPRINT ARXIV:1701.06420, 2017. Proceedings... [S.1.:s.n.], 2017.

BINKERT, N. et al. The gem5 simulator. In: SIGARCH COMPUT. ARCHIT. NEWS, 2011. Proceedings... New York, NY, USA: ACM, 2011. p. 1-7.

EBRAHIMI, E. et al. Fairness via source throttling: a configurable and high-performance fairness substrate for multi-core memory systems. In: ACM SIGPLAN NOTICES, 2010. Proceedings... New York, USA: ACM, 2010. p. 335-346.

ELLIOTT, D. G. et al. Computational ram: Implementing processors in memory. In: IEEE DESIGN AND TEST OF COMPUTERS, 1999. Proceedings... [S.1.]: IEEE, 1999. p. 32-41.

FARMAHINI-FARAHANI, A. et al. Drama: an architecture for accelerated processing near memory. In: IEEE COMPUTER ARCHITECTURE LETTERS, 2014. Proceedings... [S.1.]: IEEE, 2014. p. 26-29.

FARMAHINI-FARAHANI, A. et al. Nda: Near-dram acceleration architecture leveraging commodity dram devices and standard memory modules. In: 21ST INTERNATIONAL SYMPOSIUM ON HIGH PERFORMANCE COMPUTER ARCHITECTURE (HPCA), 2015. Proceedings... [S.1.]: IEEE, 2015. p. 283-295. 
GHENASSIA, F. et al. Transaction-level modeling with SystemC. [S.1.]: Springer, 2005.

HASSAN, H. et al. Softmc: A flexible and practical open-source infrastructure for enabling experimental dram studies. In: IEEE INTERNATIONAL SYMPOSIUM ON HIGH PERFORMANCE COMPUTER ARCHITECTURE (HPCA), 2017. Proceedings... [S.1.]: IEEE, 2017. p. 241-252.

HONG, D. U. L. et. al. S. 25.2 a $1.2 \mathrm{v} 8 \mathrm{gb} 8$-channel $128 \mathrm{gb} / \mathrm{s}$ high-bandwidth memory (hbm) stacked dram with effective microbump i/o test methods using 29nm process and tsv. In: IEEE INTERNATIONAL SOLID-STATE CIRCUITS CONFERENCE DIGEST OF TECHNICAL PAPERS (ISSCC), 2014. Proceedings... [S.1.]: IEEE, 2014. p. 432-433.

HSIEH, K. et al. Accelerating pointer chasing in 3d-stacked memory: Challenges, mechanisms, evaluation. In: IEEE 34TH INTERNATIONAL CONFERENCE ON COMPUTER DESIGN (ICCD), 2016. Proceedings... [S.1.]: IEEE, 2016. p. 25-32.

Hybrid Memory Cube Consortium. Hybrid Memory Cube Specification Rev. 2.0. 2013. Http://www.hybridmemorycube.org/.

JACOB, B.; NG, S.; WANG, D. Memory systems: cache, DRAM, disk. [S.1.]: Morgan Kaufmann, 2010.

JAYADEVAPPA, S.; SHANKAR, R.; MAHGOUB, I. A comparative study of modelling at different levels of abstraction in system on chip designs: a case study. In: IEEE COMPUTER SOCIETY ANNUAL SYMPOSIUM ON VLSI, 2004. Proceedings... [S.1.]: IEEE, 2004. p. 52-58.

JEON, D. I.; CHUNG, K. S. Cashmc: A cycle-accurate simulator for hybrid memory cube. In: IEEE COMPUTER ARCHITECTURE LETTERS, 2016. Proceedings... [S.1.]: IEEE, 2016. p. 1-1.

LEE, C. J. et al. Dram-aware last-level cache writeback: Reducing write-caused interference in memory systems. HPS Technical Report, TR-HPS-2010-002, 2010.

LEE, D. et al. Simultaneous multi-layer access: Improving 3d-stacked memory bandwidth at low cost. ACM Transactions on Architecture and Code Optimization (TACO), ACM, v. 12, n. 4, p. 63, 2016.

LEE, D. et al. Adaptive-latency dram: Optimizing dram timing for the common-case. In: 21ST INTERNATIONAL SYMPOSIUM ON HIGH PERFORMANCE COMPUTER ARCHITECTURE (HPCA), 2015. Proceedings... [S.1.]: IEEE, 2015. p. 489-501.

LEIDEL, J. D.; CHEN, Y. Hmc-sim-2.0: A simulation platform for exploring custom memory cube operations. In: IEEE INTERNATIONAL PARALLEL AND DISTRIBUTED PROCESSING SYMPOSIUM WORKSHOPS (IPDPSW), 2016. Proceedings... [S.1.]: IEEE, 2016. p. 621-630.

LI, S. et al. The McPAT Framework for Multicore and Manycore Architectures: Simultaneously Modeling Power, Area, and Timing. Transactions on Architecture and Code Optimization, v. 10, n. 1, p. 5, 2013. 
LIM, K. et al. Disaggregated memory for expansion and sharing in blade servers. In: ACM SIGARCH COMPUTER ARCHITECTURE NEWS, 2009. Proceedings... New York, USA: ACM, 2009. p. 267-278.

LIU, J. et al. Disaggregated memory for expansion and sharing in blade servers. In: ACM SIGARCH COMPUTER ARCHITECTURE NEWS, 2013. Proceedings... New York, USA: ACM, 2013. p. 60-71.

LIU, J. et al. Raidr: Retention-aware intelligent dram refresh. In: ACM SIGARCH COMPUTER ARCHITECTURE NEWS, 2012. Proceedings... New York, USA: ACM, 2012. p. 1-12.

MATHEW, D. et al. A bank-wise dram power model for system simulations. In: WORKSHOP ON: RAPID SIMULATION SIMULATION AND PERFORMANCE EVALUATION: METHODS AND TOOLS (RAPIDO), 2017, Stockholm, Sweden. Proceedings... [S.1.:s.n], 2017.

MOSCIBRODA, T.; MUTLU, O. Memory performance attacks: Denial of memory service in multi-core systems. In: 16TH USENIX SECURITY SYMPOSIUM ON USENIX SECURITY SYMPOSIUM, 2007. Proceedings... [S.1.]: USENIX Association, 2007.

MURPHY, R. On the effects of memory latency and bandwidth on supercomputer application performance. In: 10TH INTERNATIONAL SYMPOSIUM ON WORKLOAD CHARACTERIZATION (IISWC), 2007. Proceedings... [S.l.]: IEEE, 2007. p. 35-43.

NAIR, R. et al. Active memory cube: A processing-in-memory architecture for exascale systems. IBM Journal of Research and Development, v. 59, n. 2/3, March 2015.

OLIVEIRA, G. F. et al. Nim: An hmc-based machine for neuron computation. In: INTERNATIONAL SYMPOSIUM ON APPLIED RECONFIGURABLE COMPUTING, 2017, Delft, Netherlands. Proceedings... Cham, Switzerland: Springer, 2017. p. 28-35.

PANDA, P. R. Systemc-a modeling platform supporting multiple design abstractions. In: 14TH INTERNATIONAL SYMPOSIUM ON SYSTEM SYNTHESIS, 2001. Proceedings... [S.1.]: IEEE, 2001. p. 75-80.

PATEL, M.; KIM, J. S.; MUTLU, O. The reach profiler (reaper): Enabling the mitigation of dram retention failures via profiling at aggressive conditions. In: 44TH ANNUAL INTERNATIONAL SYMPOSIUM ON COMPUTER ARCHITECTURE, 2017. Proceedings... New York, USA: ACM, 2017. p. 255-268.

PATTERSON, D. et al. A case for intelligent ram. IEEE Micro, v. 17, n. 2, p. 34-44, Mar 1997. ISSN 0272-1732.

PUGSLEY, S. et al. Comparing Implementations of Near-Data Computing with In-Memory MapReduce Workloads. IEEE Micro, v. 34, n. 4, p. 44-52, July 2014.

RADULOVIC, M. et al. Another trip to the wall: How much will stacked dram benefit hpc? In: INTERNATIONAL SYMPOSIUM ON MEMORY SYSTEMS, 2015. Proceedings... [New York, USA]: ACM, 2015. p. 31-36. 
ROSENFELD, P. Performance exploration of the hybrid memory cube. Thesis (PhD) — University of Maryland, 2014.

ROSENFELD, P.; COOPER-BALIS, E.; JACOB, B. Dramsim2: A cycle accurate memory system simulator. IEEE Computer Architecture Letters, IEEE, v. 10, n. 1, p. 16-19, 2011.

SANTOS, P. C. et al. Exploring cache size and core count tradeoffs in systems with reduced memory access latency. In: 24TH EUROMICRO INTERNATIONAL CONFERENCE ON PARALLEL, DISTRIBUTED, AND NETWORK-BASED PROCESSING (PDP), 2016. Proceedings... [S.1.]: IEEE, 2016. p. 388-392.

SANTOS, P. C. et al. Operand size reconfiguration for big data processing in memory. In: DESIGN, AUTOMATION AND TEST IN EUROPE CONFERENCE (DATE), 2017, Lausanne, Switzerland. Proceedings... [S.1.]: IEEE, 2017.

SCHALLER, R. R. Moore's law: past, present and future. IEEE Spectrum, v. 34, n. 6, p. 52-59, Jun 1997. ISSN 0018-9235.

SESHADRI, V. et al. Rowclone: Fast and energy-efficient in-dram bulk data copy and initialization. In: 46TH ANNUAL IEEE/ACM INTERNATIONAL SYMPOSIUM ON MICROARCHITECTURE (MICRO), 2013. Proceedings... [S.l.]: IEEE, 2013. p. 185-197.

SHIVAKUMAR, P.; JOUPPI, N. P. Cacti 3.0: An integrated cache timing, power, and area model. Technical Report 2001/2, Compaq Computer Corporation, 2001.

STUECHELI, J. et al. The virtual write queue: Coordinating dram and last-level cache policies. In: ACM. ACM SIGARCH Computer Architecture News. [S.1.], 2010. v. 38, n. 3, p. 72-82.

SUBRAMANIAN, L. et al. Mise: Providing performance predictability and improving fairness in shared main memory systems. In: IEEE 19TH INTERNATIONAL SYMPOSIUM ON HIGH PERFORMANCE COMPUTER ARCHITECTURE (HPCA), 2013. Proceedings... [S.1.]: IEEE, 2013. p. 639-650.

SURA, Z. et al. Data access optimization in a processing-in-memory system. In: PROCEEDINGS OF THE 12TH ACM INTERNATIONAL CONFERENCE ON COMPUTING FRONTIERS, 2015. Proceedings... New York, USA: ACM, 2015.

Tezzaron. DiRAM4 - 3D Memory. 2015. Https://tezzaron.com/products/diram4-3dmemory/.

WULF, W. A.; MCKEE, S. A. Hitting the memory wall: Implications of the obvious. SIGARCH Comput. Archit. News, ACM, New York, NY, USA, v. 23, n. 1, p. 20-24, mar. 1995. ISSN 0163-5964. Available from Internet: $<$ http://doi.acm.org/10.1145/216585.216588>.

XI, S. L. et al. Quantifying sources of error in mcpat and potential impacts on architectural studies. In: 21ST INTERNATIONAL SYMPOSIUM ON HIGH PERFORMANCE COMPUTER ARCHITECTURE (HPCA), 2015. Proceedings... [S.1.]: IEEE, 2015. p. $577-589$. 
XU, L.; ZHANG, D. P.; JAYASENA, N. Scaling deep learning on multiple in-memory processors. In: WONDP: 3RD WORKSHOP ON NEAR-DATA PROCESSING IN CONJUNCTION WITH MICRO-48, 2015. Proceedings... [S.1.:s.n], 2015.

YOO, J. et al. Vssim: Virtual machine based ssd simulator. In: 29TH SYMPOSIUM ON MASS STORAGE SYSTEMS AND TECHNOLOGIES (MSST), 2013. Proceedings... [S.1.]: IEEE, 2013. p. 1-14. 